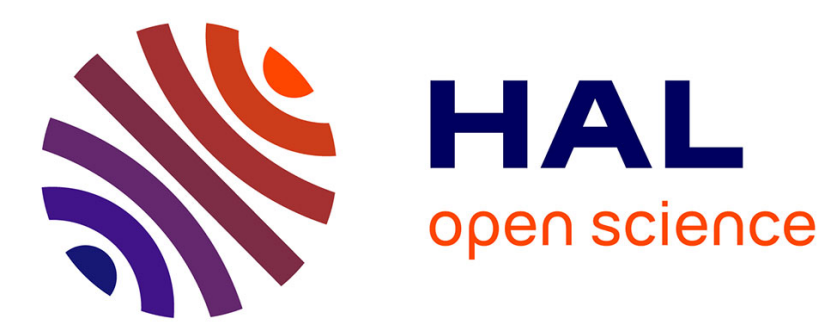

\title{
Simulation and modelling of the waves transmission and generation in a stator blade row in a combustion-noise framework
}

\author{
Matthieu Leyko, Ignacio Duran, Stphane Moreau, Franck Nicoud, Thierry \\ Poinsot
}

\section{To cite this version:}

Matthieu Leyko, Ignacio Duran, Stphane Moreau, Franck Nicoud, Thierry Poinsot. Simulation and modelling of the waves transmission and generation in a stator blade row in a combustion-noise framework. Journal of Sound and Vibration, 2014, 333 (23), pp.6090 - 6106. 10.1016/j.jsv.2014.06.034 . hal-01101467

\section{HAL Id: hal-01101467 \\ https://hal.science/hal-01101467}

Submitted on 8 Jan 2015

HAL is a multi-disciplinary open access archive for the deposit and dissemination of scientific research documents, whether they are published or not. The documents may come from teaching and research institutions in France or abroad, or from public or private research centers.
L'archive ouverte pluridisciplinaire HAL, est destinée au dépôt et à la diffusion de documents scientifiques de niveau recherche, publiés ou non, émanant des établissements d'enseignement et de recherche français ou étrangers, des laboratoires publics ou privés. 


\title{
Simulation and modelling of the waves transmission and generation in a stator blade row in a combustion-noise framework
}

\author{
Matthieu Leyko $^{\mathrm{b}}$, Ignacio Duran ${ }^{\mathrm{a}, 1}$, Stphane Moreau $^{\mathrm{c}}$, Franck Nicoud ${ }^{\mathrm{d}}$, Thierry Poinsot ${ }^{\mathrm{e}}$ \\ ${ }^{a}$ CERFACS, 31057 Toulouse, France \\ ${ }^{b}$ Snecma, 77550 Moissy-Cramayel, France \\ ${ }^{c}$ Université de Sherbrooke, Sherbrooke, QC J1K2R1, Canada \\ ${ }^{d}$ I3M, Université Montpellier II, UMR CNRS 5149, France \\ e Institut de Mécanique des Fluides de Toulouse, 31400 Toulouse, France
}

\begin{abstract}
The combustion noise in aero-engines is known to have two different origins. First, the direct combustion noise is directly generated by the flame itself. Second, the indirect combustion noise is caused by the acceleration in the turbine stages, of entropy spots generated by the combustion. In both cases, the turbo-machinery is involved in the combustion-noise transmission and generation. Numerical simulations are performed in the present study to assess the global noise for a real aeronautical configuration. On the one hand, the acoustic and entropy transfer functions of an isolated blade row are obtained using two-dimensional unsteady simulations. The transfer functions of the blade row are compared with the model of Cumpsty and Marble that assumes an axially compact configuration. On the other hand, the acoustic and entropy sources coming from a combustion chamber are calculated from a threedimensional Large Eddy Simulation (LES). This allows an evaluation of the error introduced by the model for the present combustion chamber using the previous numerical simulations. A significant error is found for the indirect combustion noise, whereas it stays reasonable for the direct one.
\end{abstract}

Keywords: Aeroacoustics, Combustion noise

\section{Introduction}

A significant part of the noise emitted from aero-engines is generated by combustion. The relative importance of this source is expected to increase with the next generations of engines where both fan and jet noise are reduced with 
such technologies as chevrons, micro-jets or highly-swept and leaned composite blades. In the seventies, Candel [3], Marble and Candel [17] and Cumpsty and Marble [6] showed that combustion noise could be both direct or indirect. Direct combustion noise comes from the noise emitted by the turbulent flame within the combustion chamber, and is then transmitted and reflected through the upstream (compressor) and downstream (turbine) turbo-machinery stages. Indirect combustion noise comes from entropy fluctuations generated by the flame that produce noise when passing through the downstream turbine stages. This noise source is caused by the conversion of entropy spots into acoustic waves when they are accelerated in the blade rows. Therefore, indirect noise becomes relevant for large variations of Mach numbers. Candel [3] showed that this source could be important in aero-engines and could explain the observed excess noise. Cumpsty and Marble [5] also confirmed these results with an analytical model [6]. More recently, Leyko et al. [16] obtained scaling laws for a simplified one-dimensional (1-D) model combustor, which showed that indirect combustion noise could even be ten times larger than the direct one. However, in practice both sources must be taken into account. Indeed on the one hand, the noise generation caused by entropy fluctuations passing through the turbine stages, and the transmission of the noise generated within the combustion chamber have to be managed correctly to evaluate the noise at the engine outlet. On the other hand, the acoustic reflection by the compressor stages has to be considered to predict the impedance of the combustion chamber properly. In both cases, waves transmission and generation through several blade rows are involved, and the coupling within all stages must be considered. More specifically, the transfer functions of the turbo-machinery stages for the waves involved in the combustion process (acoustic, entropy and vortical) must be calculated.

An important number of analytical and semi-analytical models have been proposed for one- (1-D), two- (2-D) and three-dimensional (3-D) flows to deal with the propagation of acoustic and vortical waves through the turbo-machinery stages, ranging from simplest 1-D model dealing with compact nozzle to the most sophisticated ones involving 3-D blades. Tsien [28] and Crocco [4] first proposed an approach to calculate propagation of acoustic waves in 1-D nozzles. Muir $[21,22]$ then used the actuator-disk theory that replaces the actual 2-D configurations with an infinitely thin blade row, and classical conservation laws to establish 2-D relations between upstream and downstream flow. 
Kaji and Okazaki [12] first proposed a semi actuator-disk theory assuming that the spacing between the blades was infinitely thin but that the chord-length was finite. They then extended the model to a finite chord-length and a finite spacing between blades [13]. For both cases, the equations are solved numerically. Many other models are available for such 2-D problems. Recently Posson and Roger [25] proposed a three-dimensional rectilinear cascade model both for generation and transmission losses based on Glegg's response [25], which also accounted for cascade effects and finite chord, but neglected flow deviation (flat plates at zero angle of incidence). A generalization of this model to a more realistic annular cascade has also been presented [24].

The number of models dealing with entropy waves is smaller. In the seventies, Marble and Candel [17] following the work of Tsien [28] and Crocco [4], developed an approach taking into account acoustic and entropy waves for 1-D compact nozzles. At the same time, Ffwocs-Williams and Howe [10] developed a Green's function to propagate entropy waves in non-compact 1-D nozzles, but with a low-Mach number assumption. Pickett [23] established relationships based on mass and momentum conservation to propagate entropy waves in a 2-D compact blade row. Extending the approach of Marble and Candel, Cumpsty and Marble also proposed a model based on the linearized conservation equations of mass and total enthalpy, to deal with waves transmission and generation (involving entropy waves) through an axially compact blade row in a 2-D periodic and uniform inviscid flow. Finally Stow and Dowling [27] studied circumferential modes in choked nozzles numerically.

Bake et al. [1] have investigated the indirect noise for a 1-D nozzle flow experimentally, in the so-called Entropy Wave Generator (EWG). Mühlbauer et al. [20], Leyko et al. [15] and Duran et al. [9] have simulated this configuration for several flow conditions (subsonic and supersonic choked nozzle), and have reproduced the measured indirect and direct noise with acceptable precision. Leyko et al. [15] have evidenced the critical effect of the outlet reflections in the supersonic choked case. They have also shown that the 1-D theory of Marble and Candel [17] works very well for this nozzle and flow configuration, and it could help designing future experimental set-ups to limit the effect of downstream acoustic impedance. Duran et al. [9] have shown similar results for the low subsonic cases provided both the direct noise from the entropy generator and the inlet reflections are also considered. Beyond a throat Mach number of 0.3 , the 
nozzle becomes non-compact and this new effect should be accounted for to reproduce the measurements. However, no experimental or numerical studies have been proposed yet, to investigate indirect noise for a 2-D flow within a blade row. Experimental measurements of such phenomenon remain nowadays difficult on real engines because of both the compactness of the turbo-machinery stages, and of the high operating pressures and temperatures. What is proposed in the present study instead is to look at the theoretical aspects of Cumpsty and Marble's extension [6] of Marble and Candel's model [17], and to assess its validity by comparing with dedicated numerical results. The simplest passage of entropic waves and acoustic waves through an isolated stator vane is thus studied both numerically and analytically, extending the work of Mishra and Bodony [18]. The theoretical aspects of the analytical model are first outlined in Sec. 2, whereas the propagation of entropy, vorticity (because of the two-dimensional flow) and acoustic waves through an isolated blade is addressed with a Large-Eddy-Simulation (LES) code in Sec. 3. Finally, the results are presented in Sec. 4 and conclusions about the validity of the model are drawn in Sec. 5.

\section{Theory}

This section provides a detailed description of the model of Cumpsty and Marble [6] for the propagation of waves through a stator blade, that has been subsequently used in a complete wave-propagation model within a turboengine [7]. This model is based on the same principles as the compact nozzle of Marble and Candel [17], but it assumes a 2-D configuration to take into account the circumferential component of the turbomachinery and the flow deflection inducing vorticity fluctuations. The radial component of the flow is neglected, which is valid for large hub-to-tip ratio and perfect radial equilibrium in the turbomachine. The model assumes that the blade row is axially compact and that the blades spacing is small compared to the chord. The wave lengths of the different waves involved in the process are thus large compared with the axial length of the blade row. The steady flows on both sides of the blade row are assumed to be different, but uniform, so that the former is treated as a planar interface, as shown in Fig. 1. Assuming that the perturbations are small, balance equations can be written on both sides, the outgoing waves can be evaluated as a function of the incoming ones and of the main flow parameters. The primitive variables of the flow considered 

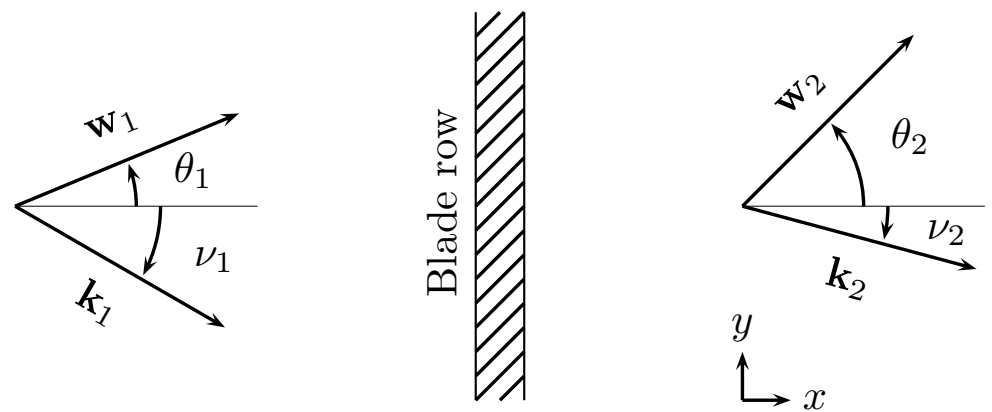

Figure 1: Description of the model approach. $\mathbf{w}$ is the velocity vector and $\mathbf{k}$ is the wave vector.

here are the pressure $p$, the density $\rho$, the relative velocity vector $\mathbf{w}$ and the flow angle $\theta$. Similarly, the waves are characterized by the wave vector $\mathbf{k}$ and the wave angle $v$.

As a first step, let's consider the propagation of the perturbations in the upstream and downstream regions where the flow is assumed inviscid and uniform. The linearized Euler equations (LEE) in a steady uniform flow are given by,

$$
\begin{gathered}
\frac{D}{D t}\left(\frac{\rho^{\prime}}{\rho}\right)+\frac{\partial u^{\prime}}{\partial x}+\frac{\partial v^{\prime}}{\partial y}=0, \\
\frac{D}{D t}\left(u^{\prime}\right)=-\frac{1}{\rho} \frac{\partial p^{\prime}}{\partial x}, \\
\frac{D}{D t}\left(v^{\prime}\right)=-\frac{1}{\rho} \frac{\partial p^{\prime}}{\partial y}, \\
\frac{D}{D t}\left(\frac{s^{\prime}}{c_{p}}\right)=\frac{D}{D t}\left(\frac{p^{\prime}}{\gamma p}-\frac{\rho^{\prime}}{\rho}\right)=0,
\end{gathered}
$$

where the material derivative is written as

$$
\frac{D}{D t}=\frac{\partial}{\partial t}+\mathbf{W} \cdot \nabla=\frac{\partial}{\partial t}+U \frac{\partial}{\partial x}+V \frac{\partial}{\partial y}
$$

with $U=W \cos \theta$ and $V=W \sin \theta$ the axial and circumferential components of the mean velocity. The fluctuating variables $u^{\prime}$ and $v^{\prime}$ are the velocity perturbations in the $x$ and $y$ directions respectively. They can be related to $w^{\prime}$ and $\theta^{\prime}$ through the velocity triangles 


$$
\frac{u^{\prime}}{U}=\frac{w^{\prime}}{W}-\theta^{\prime} \tan \theta, \quad \frac{v^{\prime}}{V}=\frac{w^{\prime}}{W}+\frac{\theta^{\prime}}{\tan \theta} .
$$

In each uniform region any wave $\phi$ is assumed harmonic and can be written in the form

$$
w^{\phi}=A_{\phi} \exp \left[i\left(\omega t-\mathbf{k}_{\phi} \cdot \mathbf{x}\right)\right]
$$

where $A_{\phi}$ represents the amplitude, $\omega$ the angular frequency and $\mathbf{k}_{\phi}$ the wave vector associated with the wave, which can be expressed as a combination of the axial and circumferential components,

$$
\mathbf{k}_{\phi} \cdot \mathbf{x}=k_{x, \phi} x+k_{y, \phi} y, \quad \text { with } \quad k_{x, \phi}=k_{\phi} \cos v_{\phi} \quad \text { and } \quad k_{y, \phi}=k_{\phi} \sin v_{\phi}
$$

$k_{\phi}$ is the modulus of the wave vector $\mathbf{k}_{\phi}$. The latter is scaled with the mean sound speed $c$ and the angular frequency, yielding the dimensionless wave vector, $\mathbf{K}_{\phi}=\mathbf{k}_{\phi} c / \omega$. The material derivative given by Eq. (5) then becomes

$$
\frac{D}{D t}=i \omega-U i k_{x, \phi}-V i k_{y, \phi}
$$

For instance, the dimensionless entropy fluctuation $s^{\prime} / c_{p}$ is defined as

$$
w^{s} \equiv \frac{s^{\prime}}{c_{p}}=A_{s} \exp \left[i\left(\omega t-\mathbf{k}_{s} \cdot \mathbf{x}\right)\right]
$$

where $s^{\prime}$ is the entropy fluctuation and $c_{p}$ the thermal capacity at constant pressure. Inserting Eq. (10) into Eq. (4) yields the following dispersion equation for the entropy wave

$$
\omega-W \cos (\theta) k_{s} \cos \left(v_{s}\right)-W \sin (\theta) k_{s} \sin \left(v_{s}\right)=0 .
$$

If the dimensionless wave number $K_{s}=k_{s} c / \omega$ is introduced, the dispersion equation can be rearranged as follows

$$
K_{S} M \cos \left(v_{s}-\theta\right)=1,
$$

where $M$ is the steady Mach number based on the steady velocity magnitude $W$ and the steady speed of sound $c$. By definition, the entropy wave does not generate any pressure or velocity perturbation. Therefore, the fluctuations of 
primitive variables $\left(s^{\prime} / c_{p}, w^{\prime} / c, p^{\prime} / \gamma p\right.$ and $\left.\theta^{\prime}\right)$ generated by the entropy wave are

$$
\left\{\begin{array}{c}
s_{s}^{\prime} / c_{p} \\
w_{s}^{\prime} / c \\
p_{s}^{\prime} / \gamma p \\
\theta_{s}^{\prime}
\end{array}\right\}=\left\{\begin{array}{c}
1 \\
0 \\
0 \\
0
\end{array}\right\} w^{s} .
$$

Similarly, the perturbation of vorticity $\xi^{\prime} \equiv \partial v^{\prime} / \partial x-\partial u^{\prime} / \partial y$ is written as follows

$$
w^{v} \equiv \frac{\xi^{\prime}}{\omega}=A_{\xi} \exp \left[i\left(\omega t-\mathbf{k}_{\xi} \cdot \mathbf{x}\right)\right]
$$

Taking the spatial derivatives of Eq. (2) and (3) leads to

$$
\begin{gathered}
\frac{\partial^{2} u^{\prime}}{\partial t \partial y}+U \frac{\partial^{2} u^{\prime}}{\partial x \partial y}+V \frac{\partial^{2} u^{\prime}}{\partial y \partial y}=-\frac{1}{\rho} \frac{\partial^{2} p^{\prime}}{\partial x \partial y} \\
\frac{\partial^{2} v^{\prime}}{\partial t \partial x}+U \frac{\partial^{2} v^{\prime}}{\partial x \partial x}+V \frac{\partial^{2} v^{\prime}}{\partial y \partial x}=-\frac{1}{\rho} \frac{\partial^{2} p^{\prime}}{\partial y \partial x}
\end{gathered}
$$

Subtracting both relations in Eq. (15) yields

$$
\frac{\partial}{\partial t}\left(\frac{\partial v^{\prime}}{\partial x}-\frac{\partial u^{\prime}}{\partial y}\right)+U \frac{\partial}{\partial x}\left(\frac{\partial v^{\prime}}{\partial x}-\frac{\partial u^{\prime}}{\partial y}\right)+V \frac{\partial}{\partial y}\left(\frac{\partial v^{\prime}}{\partial x}-\frac{\partial u^{\prime}}{\partial y}\right)=0
$$

Finally, the vorticity fluctuation verifies the following relationship

$$
\frac{D}{D t}\left(\xi^{\prime}\right)=0
$$

By similarity with the entropy fluctuations, the dimensionless vorticity wave number $K_{\xi}=k_{\xi} c / \omega$ checks the following dispersion equation

$$
K_{\xi} M \cos \left(v_{\xi}-\theta\right)=1 .
$$

Because vorticity waves are divergence free,

$$
\frac{\partial u_{\xi}^{\prime}}{\partial x}+\frac{\partial v_{\xi}^{\prime}}{\partial y}=0
$$


the following relationship is obtained

$$
v_{\xi}^{\prime}=-u_{\xi}^{\prime} \frac{k_{x, \xi}}{k_{y, \xi}} .
$$

By definition

$$
\xi^{\prime}=-i k_{x, \xi} v_{\xi}^{\prime}+i k_{y, \xi} u_{\xi}^{\prime}
$$

that leads to

$$
\begin{aligned}
& \frac{u_{\xi}^{\prime}}{c}=-i \frac{\xi^{\prime}}{\omega} \frac{\sin v_{\xi}}{K_{\xi}} \\
& \frac{v_{\xi}^{\prime}}{c}=+i \frac{\xi^{\prime}}{\omega} \frac{\cos v_{\xi}}{K_{\xi}}
\end{aligned}
$$

Combining Eq. (22) with Eq. (6) for the fluctuations of the velocity components $u_{\xi}^{\prime}$ and $v_{\xi}^{\prime}$ yields the corresponding fluctuations for $w_{\xi}^{\prime}$ and $\theta_{\xi}^{\prime}$

$$
\begin{aligned}
\theta_{\xi}^{\prime} & =\left(-u_{\xi}^{\prime} \sin \theta+v_{\xi}^{\prime} \cos \theta\right) / W \\
w_{\xi}^{\prime} & =u_{\xi}^{\prime} \cos \theta+v_{\xi}^{\prime} \sin \theta
\end{aligned}
$$

The fluctuations related to the vorticity wave can finally be written

$$
\left\{\begin{array}{c}
s_{\xi}^{\prime} / c_{p} \\
w_{\xi}^{\prime} / c \\
p_{\xi}^{\prime} / \gamma p \\
\theta_{\xi}^{\prime}
\end{array}\right\}=\left\{\begin{array}{c}
0 \\
-i \sin \left(v_{\xi}-\theta\right) / K_{\xi} \\
0 \\
i \cos \left(v_{\xi}-\theta\right) /\left(M K_{\xi}\right)
\end{array}\right\} w^{v}
$$

The pressure perturbation $p^{\prime} / \gamma p$ is defined as follows

$$
w^{ \pm} \equiv \frac{p_{ \pm}^{\prime}}{\gamma p}=A_{ \pm} \exp \left[i\left(\omega t-\mathbf{k}_{ \pm} \cdot \mathbf{x}\right)\right]
$$

As entropy and vorticity fluctuations are described by independent fluctuations, the acoustic waves generate a fluctuating field that is irrotational and isentropic. Eqs.(1)-(4) can be combined to give the wave equation satisfied by the 
acoustic pressure field

$$
\left(\frac{D}{D t}\right)^{2}\left(\frac{p^{\prime}}{\gamma p}\right)-c^{2}\left(\frac{\partial^{2}}{\partial x^{2}}+\frac{\partial^{2}}{\partial y^{2}}\right)\left(\frac{p^{\prime}}{\gamma p}\right)=0
$$

which leads to the dispersion equation verified by the dimensionless wave vectors $K_{ \pm}=c k_{ \pm} / \omega$

$$
\left(1-K_{ \pm} M \cos \left(v_{ \pm}-\theta\right)\right)^{2}-K_{ \pm}^{2}=0 .
$$

Indeed, two wave vectors $\mathbf{k}_{+}$and $\mathbf{k}_{-}$satisfy the wave equation (26). The wave numbers $k_{y, \pm}$ are real since the domain is periodic in the azimuthal direction. The wave numbers $k_{x,+}$ and $k_{x,-}$ can be complex and read ${ }^{1}$

$$
\begin{aligned}
K_{x, \pm} & =\frac{-M \cos \theta\left(1-K_{y, \pm} M \sin \theta\right)}{1-M^{2} \cos ^{2} \theta} \\
& \pm\left(\frac{\left(1-K_{y, \pm} M \sin \theta\right)^{2}-\left(1-M^{2} \cos ^{2} \theta\right) K_{y, \pm}^{2}}{\left(1-M^{2} \cos ^{2} \theta\right)^{2}}\right)^{\frac{1}{2}}
\end{aligned}
$$

By expressing $u_{ \pm}^{\prime}$ and $v_{ \pm}^{\prime}$ from the momentum Eqs. (2) and (3), and noticing that $\frac{\rho^{\prime}}{\rho}=\frac{p^{\prime}}{\gamma p}$ when considering acoustics only, the fluctuations of primitive variables can be related to the acoustic waves $w^{ \pm}$

$$
\left\{\begin{array}{c}
s_{ \pm}^{\prime} / c_{p} \\
w_{ \pm}^{\prime} / c \\
p_{ \pm}^{\prime} / \gamma p \\
\theta_{ \pm}^{\prime}
\end{array}\right\}=\left\{\begin{array}{c}
0 \\
K_{ \pm} \cos \left(v_{ \pm}-\theta\right) /\left[1-K_{ \pm} M \cos \left(v_{ \pm}-\theta\right)\right] \\
1 \\
K_{ \pm} \sin \left(v_{ \pm}-\theta\right) /\left[M\left(1-K_{ \pm} M \cos \left(v_{ \pm}-\theta\right)\right)\right]
\end{array}\right\} w^{ \pm}
$$

The contribution of all waves $(s, v$ and \pm ) can be summed to yield the total fluctuations of the primitive variables, since the so-called waves are independent to first-order. Equations (13), (24) and (29) are then used to build the transformation matrix $\left[\mathbf{P}_{w}^{p}\right]$, which relates the fluctuations of the primitive variables to these waves:

\footnotetext{
${ }^{1} \mathrm{~A}$ mistake is present in the expression of the acoustic wave vector in the article of Cumpsty and Marble 1977
} 


$$
\left\{\begin{array}{c}
s^{\prime} / c_{p} \\
w^{\prime} / c \\
p^{\prime} / \gamma p \\
\theta^{\prime}
\end{array}\right\}=\left[\mathbf{P}_{w}^{p}\right] \cdot\left\{\begin{array}{c}
w^{s} \\
w^{v} \\
w^{+} \\
w^{-}
\end{array}\right\}
$$

with:

$$
\left[\mathbf{P}_{w}^{p}\right]=\left[\begin{array}{cccc}
1 & 0 & 0 & 0 \\
0 & -i \frac{\sin \left(\xi_{\xi}-\theta\right)}{K_{\xi}} & \frac{K_{+} \cos \left(v_{+}-\theta\right)}{1-M K_{+} \cos \left(v_{+}-\theta\right)} & \frac{K_{-} \cos \left(\nu_{-}-\theta\right)}{1-M K_{-} \cos \left(v_{-}-\theta\right)} \\
0 & 0 & 1 & 1 \\
0 & i \frac{\cos \left(v_{\xi}-\theta\right)}{M K_{\xi}} & \frac{K_{+} \sin \left(v_{+}-\theta\right)}{M\left[1-M K_{+} \cos \left(v_{+}-\theta\right)\right]} & \frac{K_{-} \sin \left(\nu_{-}-\theta\right)}{M\left[1-M K_{-} \cos \left(\nu_{-}-\theta\right)\right]}
\end{array}\right]
$$

As a second step, the blade row itself is assumed compact. Therefore the fluctuations of entropy, mass-flow and energy are the same on both sides. For small perturbations, the conservation of the entropy between the inlet and the outlet of the blade row leads to:

$$
\left(\frac{s^{\prime}}{c_{p}}\right)_{1}=\left(\frac{s^{\prime}}{c_{p}}\right)_{2}
$$

where (1) relates to the inlet of the blade row and (2) to its outlet, as shown in Fig. 1. For small perturbations, the mass-flow rate conservation can be expressed as

$$
\left(\frac{p^{\prime}}{\gamma p}\right)_{1}+\frac{1}{M_{1}}\left(\frac{w^{\prime}}{c}\right)_{1}-\theta_{1}^{\prime} \tan \theta_{1}-\left(\frac{s^{\prime}}{c_{p}}\right)_{1}=\left(\frac{p^{\prime}}{\gamma p}\right)_{2}+\frac{1}{M_{1}}\left(\frac{w^{\prime}}{c}\right)_{2}-\theta_{2}^{\prime} \tan \theta_{2}-\left(\frac{s^{\prime}}{c_{p}}\right)_{2} .
$$

The total temperature $T_{t}$ (representing the total fluid energy) is based here on the velocity magnitude $W$. Therefore, considering that $T_{t}$ is conserved through a stator vane row yields for small perturbations

$$
\begin{aligned}
\frac{1}{1+\frac{1}{2}(\gamma-1) M_{1}^{2}}\left[\left(\frac{p^{\prime}}{\gamma p}\right)_{1}+M_{1}\left(\frac{w^{\prime}}{c}\right)_{1}+\frac{1}{\gamma-1}\left(\frac{s^{\prime}}{c_{p}}\right)_{1}\right]= & \ldots \\
& \frac{1}{1+\frac{1}{2}(\gamma-1) M_{2}^{2}}\left[\left(\frac{p^{\prime}}{\gamma p}\right)_{2}+M_{1}\left(\frac{w^{\prime}}{c}\right)_{2}+\frac{1}{\gamma-1}\left(\frac{s^{\prime}}{c_{p}}\right)_{2}\right] .
\end{aligned}
$$

The axial flow upstream and downstream of the blade row is here considered to be always subsonic (like in aeroengines), therefore there are always three waves traveling downward (acoustic, entropy and vorticity) and one traveling 
upstream (acoustic). Four waves are entering the blade row (three at the inlet and one at the outlet) and four are also outgoing (one at the inlet and three at the outlet). For the flow deviation, the Kutta condition proposed by Cumpsty and Marble [6] is used

$$
\theta_{2}^{\prime}=0
$$

or in a more general form, as proposed by Cumpsty and Marble [6],

$$
\theta_{2}^{\prime}=\alpha \theta_{1}^{\prime}
$$

where $\alpha$ is a constant real number defined experimentally. Finally, Eqs. (32), (33), (34) and (36) can be written with the matrix $\left[\mathbf{E}_{p}^{e}\right]$ that relates the primitive variables fluctuations at the inlet and the outlet of the blade row:

$$
\left[\mathbf{E}_{p}^{e}\right]_{1} \cdot\left\{\begin{array}{c}
s^{\prime} / c_{p} \\
w^{\prime} / c \\
p^{\prime} / \gamma p \\
\theta^{\prime}
\end{array}\right\}_{1}=\left[\mathbf{E}_{p}^{e}\right]_{2} \cdot\left\{\begin{array}{c}
s^{\prime} / c_{p} \\
w^{\prime} / c \\
p^{\prime} / \gamma p \\
\theta^{\prime}
\end{array}\right\}_{2}
$$

with:

$$
\left[\mathbf{E}_{p}^{e}\right]_{1}=\left[\begin{array}{cccc}
1 & 0 & 0 & 0 \\
-1 & 1 / M_{1} & 1 & -\tan \theta_{1} \\
\mu_{1} /(\gamma-1) & M_{1} \mu_{1} & \mu_{1} & 0 \\
0 & 0 & 0 & \alpha
\end{array}\right]
$$

and:

$$
\left[\mathbf{E}_{p}^{e}\right]_{2}=\left[\begin{array}{cccc}
1 & 0 & 0 & 0 \\
-1 & 1 / M_{2} & 1 & -\tan \theta_{2} \\
\mu_{2} /(\gamma-1) & M_{2} \mu_{2} & \mu_{2} & 0 \\
0 & 0 & 0 & 1
\end{array}\right]
$$

where $\mu=\left(1+(\gamma-1) M^{2} / 2\right)^{-1}$. 
The overall problem of the stator row can then be expressed in terms of waves in a matrix-form as follows

$$
\underbrace{\left[\mathbf{E}_{p}^{e}\right]_{1} \cdot\left[\mathbf{P}_{w}^{p}\right]_{1}}_{[\mathbf{B}]_{1}} \cdot\left\{\begin{array}{c}
w^{s} \\
w^{v} \\
w^{+} \\
w^{-}
\end{array}\right\}_{1}^{\left[\mathbf{E}_{p}^{e}\right]_{2} \cdot\left[\mathbf{P}_{w}^{p}\right]_{2}} \cdot\left\{\begin{array}{c}
w^{s} \\
w^{v} \\
\left.w^{+}\right]_{2} \\
w^{-}
\end{array}\right\}_{2}
$$

In Eq. (40), the left-hand-side term contains the wave $w_{1}^{-}$that is travelling upward from the blade row and is unknown, whereas the other waves are imposed. The situation is reversed in the right-hand-side term, where the wave $w_{2}^{-}$is an input, and the other ones have to be found. In the case of an isolated blade row, the terms of the last column of the matrix $[\mathbf{B}]$ can be simply permuted from left-to-right and changed of sign yielding the matrices $[\mathbf{A}]_{i}$ and $[\mathbf{A}]_{o}$. Likewise the last terms of the vectors containing the waves are also permuted. Finally, the equation to be solved is

$$
\left\{\begin{array}{c}
\left(w^{s}\right)_{2} \\
\left(w^{v}\right)_{2} \\
\left(w^{+}\right)_{2} \\
\left(w^{-}\right)_{1}
\end{array}\right\}=[\mathbf{A}]_{O}^{-1} \cdot[\mathbf{A}]_{i}\left\{\begin{array}{c}
\left(w^{s}\right)_{1} \\
\left(w^{v}\right)_{1} \\
\left(w^{+}\right)_{1} \\
\left(w^{-}\right)_{2}
\end{array}\right\}
$$

The general expression of the outgoing waves is rather cumbersome. However, the present simulations shown in the next section are achieved for waves parallel to the blade row $\left(k_{y}=0\right)$, so that the expression of the outgoing waves in Eq. (41) can be simplified. Four different cases can then be defined in order to describe the problem completely and are given in Appendix A.

\section{Numerical simulation}

The goal of the numerical simulation is to calculate the actual transfer function of a turbine stator vane impinged by different perturbations, in order to evaluate the error made when using the simplified compact model of Cumpsty and Marble $[6,5]$. However, computing the turbulent flow field in the complete annular blade rows of an actual 
turbine even at a mean radius (2-D), is still out of reach. Indeed validating the model involves a very large range of characteristic times (ratio in the order of $10^{7}$ in this case). On the one hand, wavelengths of the imposed perturbations have to be sufficiently large to be able to verify the compact blade row assumption. On the other hand, the time-step of the computation has to be sufficiently small to solve the flow correctly for the small geometrical details, such as the trailing-edge for instance. The simulations are therefore done for a single 2-D stator passage as the computational domain, with periodic boundary conditions on each side of the vane to mimic an infinite blade row. As explained by Cumpsty and Marble [6], the axial Mach number through the blade rows is subsonic in most turbine applications. However, this is not the case for the global Mach number which is supersonic at the outlet of the stator vanes due to the strong deviation of the mean flow. The presence of a supersonic flow, and the shock waves downstream, adds another layer of complexity for both the numerical simulation of the propagation of waves and the analytical models. The propagation of entropy and acoustic waves through shock waves has been studied analytically $[15,11,27,8]$ and numerically [15] in 1-D configurations. The stator blade considered in this section is subsonic in order to compare the results with Cumpsty and Marbel's [6] method, which does not include the acoustic and entropy waves generated caused by the interaction of perturbations with the shock wave. Moreover, the flow at the outlet of the combustion chamber is typically turbulent. This turbulence would, if introduced in the numerical simulation, generate its own noise due to the interaction with the stator blade. As this source of noise is out of scope in the present work, no turbulence has been imposed at the inlet boundary. The 2-D flow is simulated using the unsteady compressible LES solver AVBP [19]. These Large-Eddy Simulations are performed using the Smagorinsky subgrid-scale model. The laminar and turbulent Prandtl numbers ( $\operatorname{Pr}$ and $\operatorname{Pr}_{t}$ respectively) are chosen large enough to minimize thermal diffusion within the flow $\left(\operatorname{Pr}_{t}=100\right)$, and thus to avoid problems in the interpretation of the results. The numerical method used for the simulations comes from the family of Two-step Taylor-Galerkin (TTG) proposed by Quartapelle and Selmin [26]. The selected TTG scheme is TTG4A that is third-order in space and fourth-order in time, and exhibits good dispersion properties. The topology of the computational case is sketched in Fig. 2. The mesh contains 115000 triangles and the origin of the reference frame is located at the blade trailing edge. The transfer functions are evaluated 


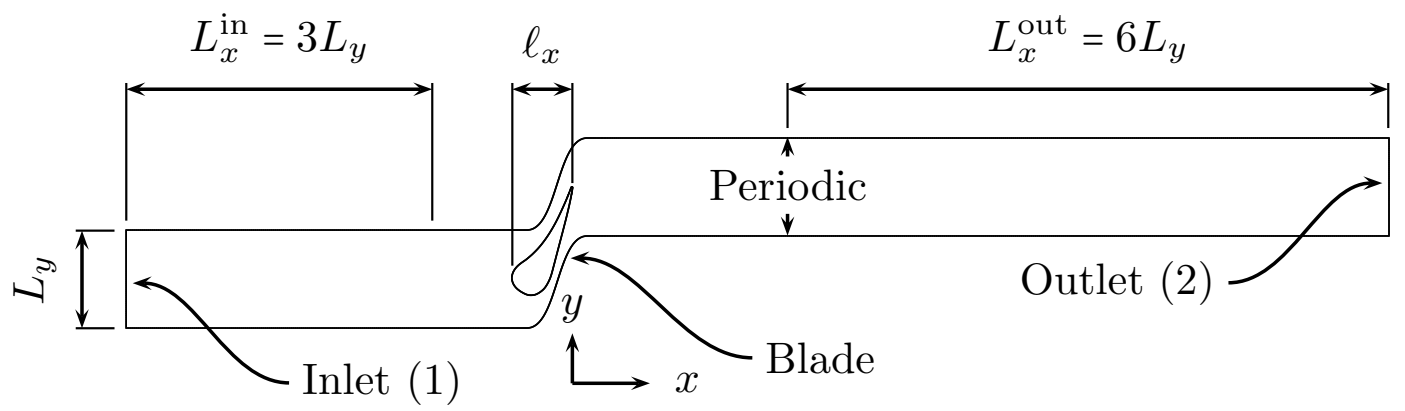

Figure 2: Description of the computational case.

by only pulsating one kind of wave (entropy, vorticity or acoustic) at a time at the corresponding computational domain boundary (inlet or outlet), whereas all the other in-going waves are set to zero. Only planar waves with a purely axial wave vector $\left(k_{y}=0\right)$ are considered. The pulsed waves are imposed in the following manner

$$
f(t)=\sum_{k=1}^{N_{k}} A_{k} \sin \left(k 2 \pi t / \tau_{0}\right)
$$

where $N_{k}$ is the number of frequencies which are pulsed (50 in the present case) and where $\tau_{0}$ is a fundamental characteristic time based on a Strouhal number of 0.1 (the former is based on the corresponding combustion chamber swirler properties). The amplitude $A_{k}$ is the same for each frequency $k$, and it is in the order of $10^{-3}$. The temporal evolution of the signal $f(t)$ is shown in Fig. 3. The spectral decomposition of this signal is of course made up of $N_{k}$ harmonic frequencies based of $1 / \tau_{0}$ and of the same magnitude. As shown in Tab. 1, four kinds of simulations are performed, which starts from a statistically converged unperturbed flow. The simulations with the perturbations of entropy (S-1) and acoustic (A-1) waves at the inlet, and with the perturbation of the acoustic wave at the outlet (A-2), provide the acoustic-to-acoustic and entropy-to-acoustic transfer functions of the blade row, whereas a fifth simulation $\mathrm{N}-0$ evaluates the global noise level, to verify that it is lower than the one produced by the perturbation of the inlet waves. In the third simulation (V-1), the vorticity-to-acoustic transfer function is calculated imposing the vorticity perturbation at the inlet. For simulations S-1 and A-1, the frequencies of the imposed signal are representative of what can be found at the outlet of a real combustion chamber. The same frequencies are used for the V- 1 and the A-2 


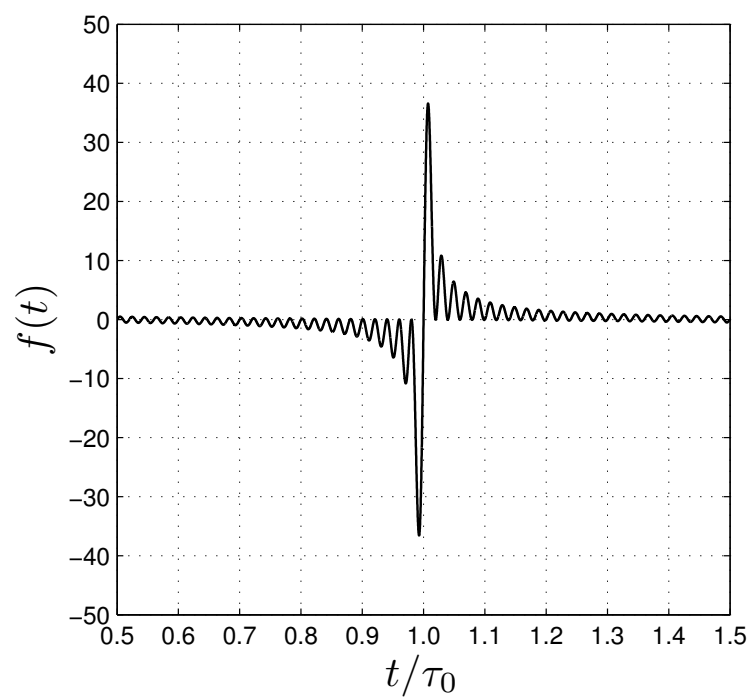

Figure 3: Temporal evolution of the pertubation imposed on acoustic or entropy waves at the computational domain inlet.

Subsonic case: $M_{1}=0.12, \theta_{1}=0$ and $M_{2}=0.66, \theta_{1}=76^{\circ}$

\begin{tabular}{cccc}
\hline Name & Pertubed wave & Location & Duration \\
\hline S-1 & Entropy & Inlet (1) & $66 \tau_{0}$ \\
V-1 & Vorticity & Inlet (1) & $66 \tau_{0}$ \\
A-1 & Acoustic & Inlet (1) & $66 \tau_{0}$ \\
A-2 & Acoustic & Outlet (2) & $66 \tau_{0}$ \\
N-0 & None & - & $18 \tau_{0}$ \\
\hline
\end{tabular}

Table 1: Description of the different simulations.

simulations. For each case a transient of $6 \tau_{0}$ is discarded and $66 \tau_{0}$ are used to collect the statistics of the simulations. 

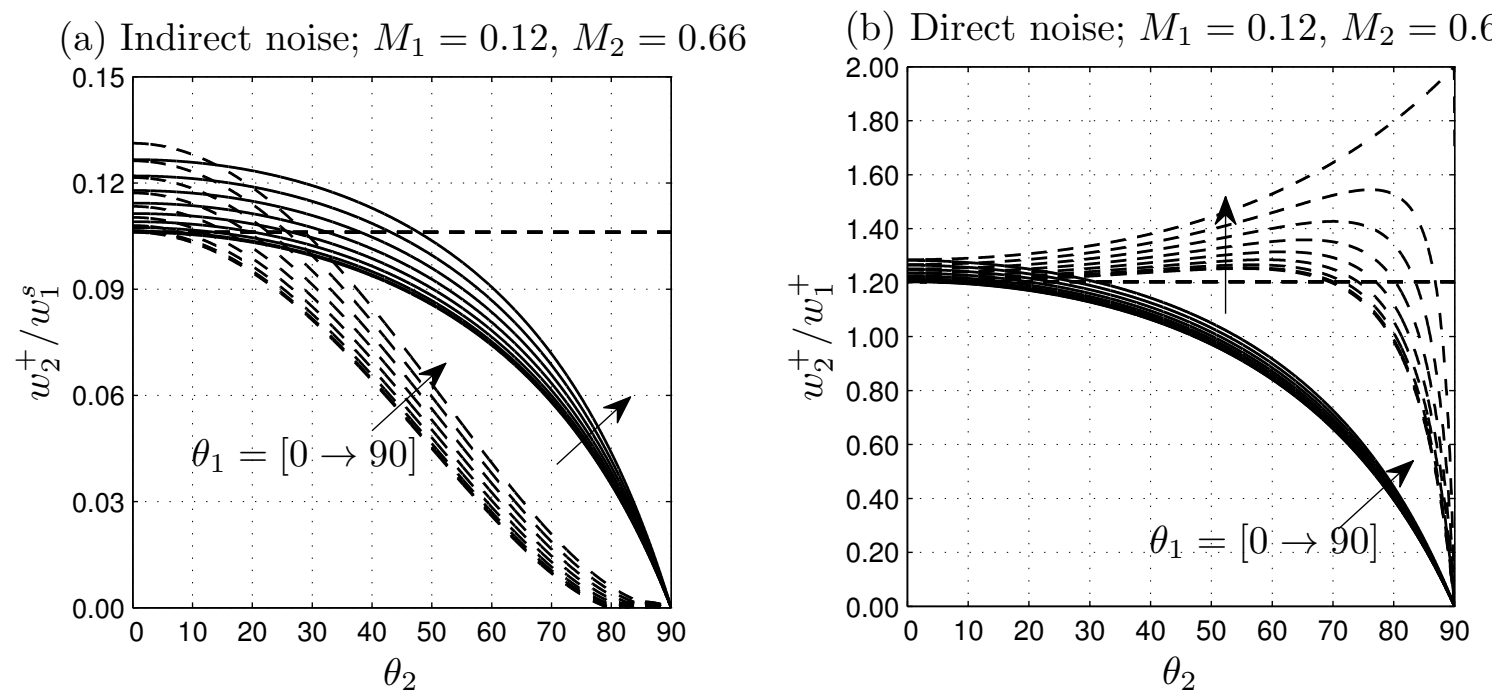

Figure 4: Acoustic response of the blade row at the outlet (2) to a perturbation at the inlet (1) for different flow directions. (a) Entropy perturbation; (b) Acoustic perturbation. Cumpsty and Marble (-), Marble and Candel with global Mach number (- - - ), Marble and Candel with axial Mach number $(---)$.

\section{Results}

As shown in Fig. 4, the generalization to a 2-D configuration (blade rows) made by Cumpsty and Marble has a major effect on the propagation of both indirect (left plot) and direct noise (right plot) compared with the 1-D model of Marble and Candel. As mentioned in Sec. 2, the 2-D model can deal with any angle of attack of the incident waves. The acoustic responses at the outlet of a blade row $w_{2}^{+}$to a purely axial entropy perturbation at the inlet $w_{1}^{s}$ (Fig. 4 (a)), and to an acoustic one at the inlet $w_{1}^{+}$(Fig. 4 (b)) are then plotted for different inlet and outlet flow angles $\theta_{1}$ and $\theta_{2}$, respectively. For the 1-D model of Marble and Candel, the Mach number based either on the total velocity magnitude or on the axial component of the velocity is used. It can be seen that the 2-D and 1-D models provide quite different results for important flow deviations that can be found in turbine stages, showing the importance of the 2-D analytical model. It should also be stressed that for $\theta_{1}=\theta_{2}=0$, the results of the two theories collapse as expected.

To understand the limitations of the 2-D analytical model, the numerical simulations are used to study the com- 
pact assumption and its influence on the transfer functions. First, only the entropy wave is imposed with the $f(t)$ signal at the inlet in the simulation S-1, and the other incoming waves are left untouched. Fig. 5 shows the field of the entropy perturbation $s^{\prime} / c_{p}$ at different times. It can be seen that the entropy wave remains planar before the blade row (there is no injection of vortical perturbations) and that it is rather perturbed after the interaction with the blade and the downstream wake. As the flow is turbulent after the blade row ( $\operatorname{Re} \approx 10^{6}$ ), all the post-processing of waves is performed by integration of the required quantities along the transversal direction, since the waves of interest are axially oriented $\left(k_{y}=0\right)$. Then, quadratic average of the waves Fourier-transform modulus is calculated along the axial direction depicted in Fig. 2. This implies that results will present only the absolute value of the waves, as the phase is lost due to the quadratic averaging. The local 1-D waves $w_{x}^{j}$ are calculated as follows:

$$
w_{x}^{j}(x, k)=\left|\frac{1}{n \tau_{0}} \int_{0}^{n \tau_{0}}\left[\frac{1}{L_{y}} \int_{0}^{L_{y}} w^{j}(x, y, t) d y\right] \exp \left(i 2 \pi k t / \tau_{0}\right) d t\right|
$$

whereas the global 1-D waves $w^{j}$ are obtained using the following relation:

$$
w^{j}(k)=\sqrt{\frac{1}{L_{x}} \int_{0}^{L_{x}}\left[w_{x}^{i}(x, k)\right]^{2} d x}
$$

In Fig. 6 the acoustic response of the blade row to an entropy perturbation is plotted as a function of the dimensionless convective wavelength $\lambda_{c} / \ell_{x}$ based on the inlet quantities. The transfer function obtained from the simulation S-1 tends to the compact model when the wavelengths are large, and decreases rapidly for small wavelengths, where the assumptions are no longer valid. Figure 7 shows the acoustic response of the blade row to an acoustic perturbation plotted as a function of the dimensionless acoustic wavelength $\lambda_{a} / \ell_{x}$ based on the inlet quantities. The frequencies in simulation A-1 are the same as in S-1, so that the wavelengths are larger than in case S-1. The transfer function obtained from simulation A-1 is thus very close to the model value since the wavelengths are large compared with the blade axial length $\ell_{x}$. As seen in Section 2, the model of Cumpsty and Marble [6] assumes that the entropy is conserved. It is obvious that the turbulent mixing in the flow downstream of the blade row strongly affects the structure of the wave and that the initial planar coherence is lost for higher frequencies as it is shown in Fig. 8. The global entropy wave in Fig. 8-a, which corresponds to the axially averaged wave, is not representative in this case, since the mixing 
(a) $t / \tau_{0}=0.1$
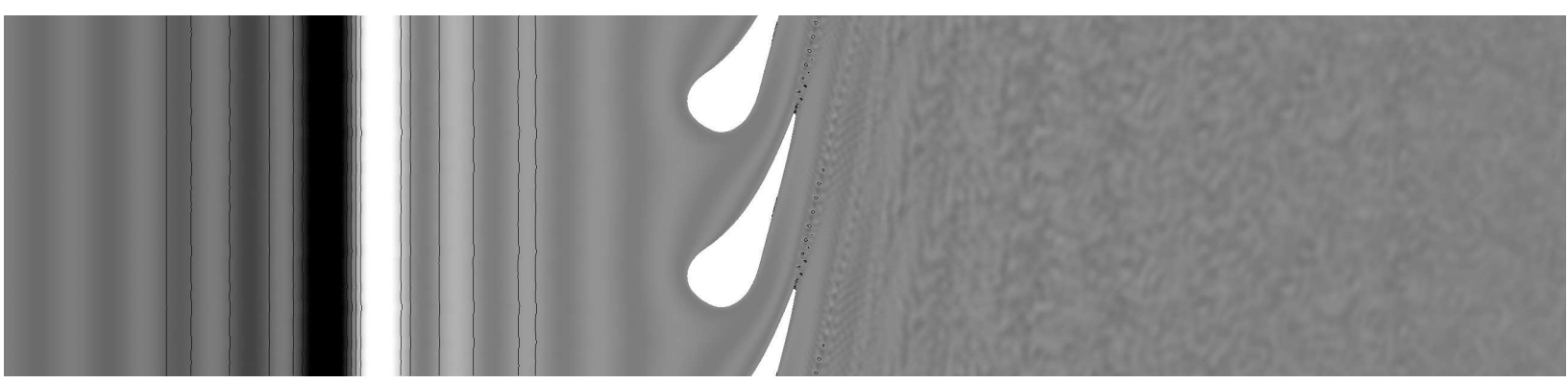

(b) $t / \tau_{0}=0.2$

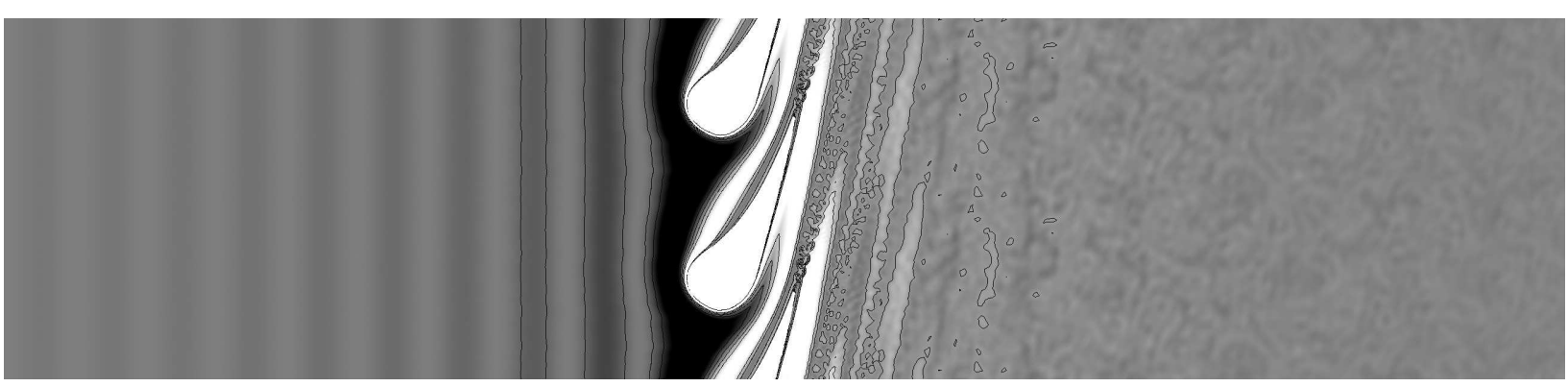

(c) $t / \tau_{0}=0.3$

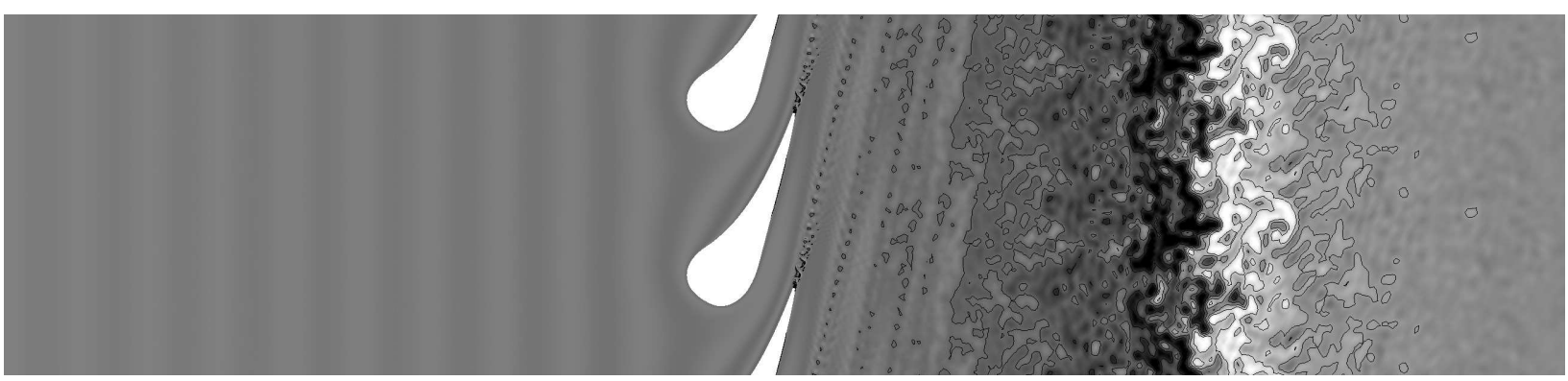

Figure 5: Instantaneous field of the entropy wave at different time steps for the case when entropy is pulsed. (a) $t / \tau_{0}=0.1$, (b) $t / \tau_{0}=0.2$, (c) $t / \tau_{0}=0.3$ 
(a)

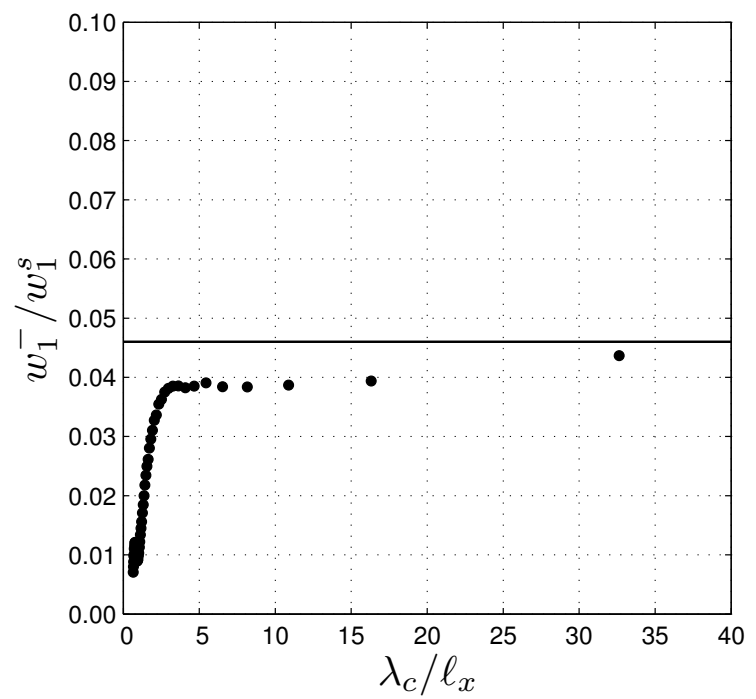

(b)

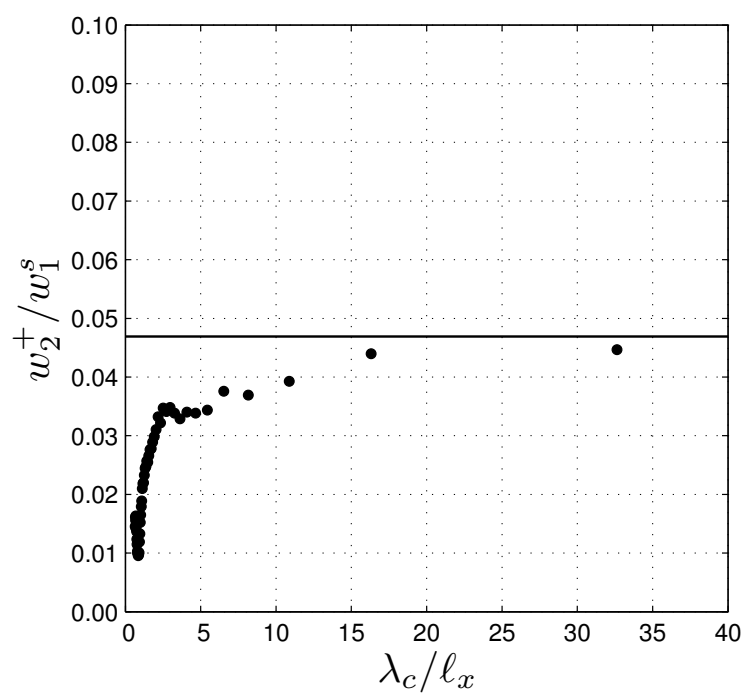

Figure 6: Acoustic response of the blade row to an entropy perturbation at the inlet (case S-1). (a) Upstream propagating waves; (b) Downstream propagating waves. Model (-), simulation (•).

(a)

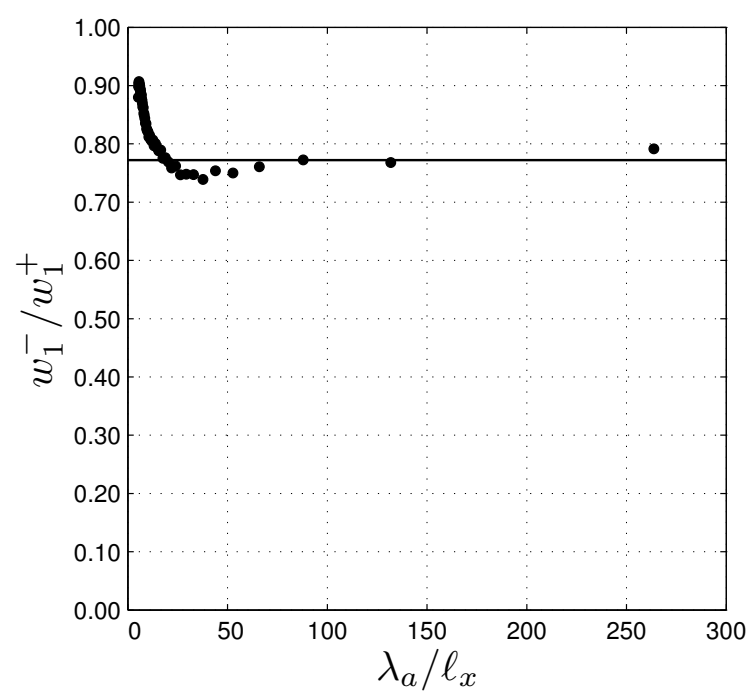

(b)

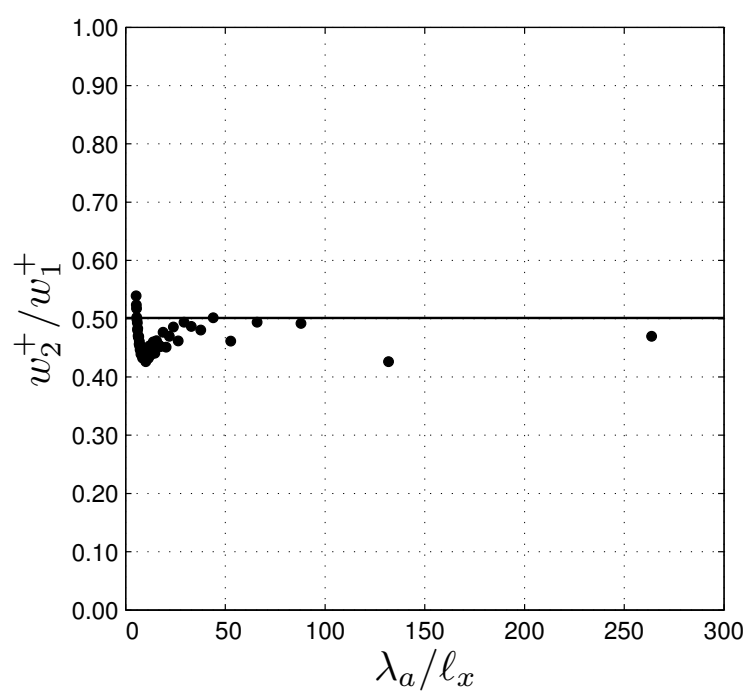

Figure 7: Acoustic response of the blade row to an acoustic perturbation at the inlet (case A-1). (a) Upstream propagating waves; (b) Downstream propagating waves. Model (-), simulation (•). 
(a)

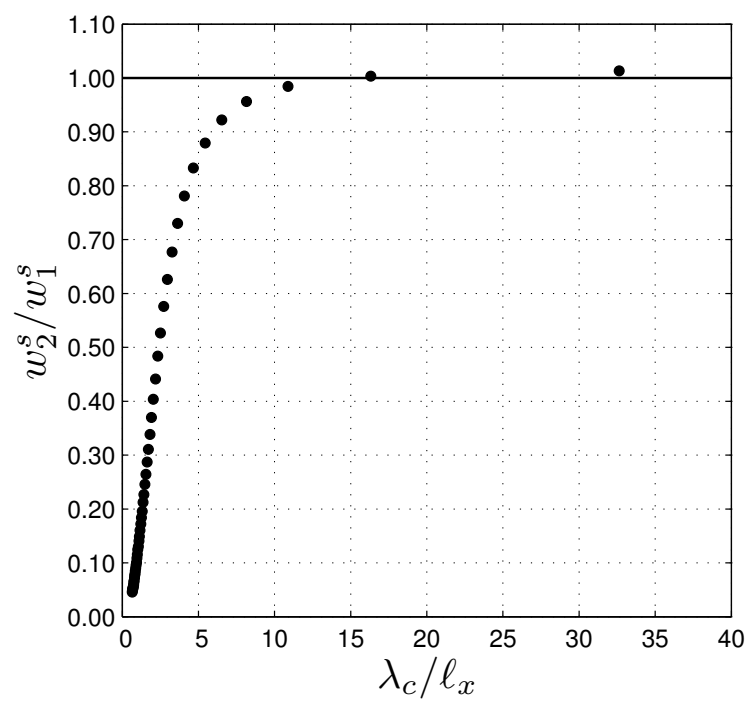

(b)

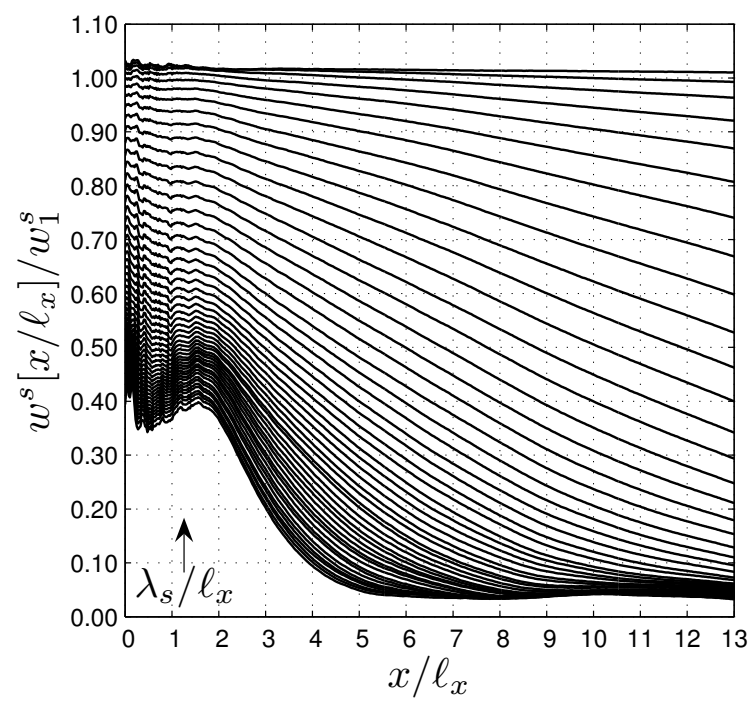

Figure 8: Entropy response of the blade row at the outlet to an entropy perturbation at the inlet. (a) Global entropy wave, where — stands for the model, and $\bullet$ for the simulation; (b) Local entropy wave at the outlet, where the different curves represent different frequencies.

process takes place after the blade row. The evolution along the axial direction of the entropy wave is more relevant of its attenuation as observed in Fig. 8-b. Large wavelengths are practically not affected by the mixing that occurs at the scales of the blade dimension, and levels remain the same until the outlet. The intensity of small wavelengths decreases more rapidly, and noticeably these waves are already attenuated at the very beginning of the downstream flow $\left(x / l_{x}=0\right)$. Since the spacing between the stator and the rotor $\Delta x$ is generally very small $\left(\Delta x / l_{x} \sim 0.1\right)$ and tends to decrease, it is interesting to understand the reasons of such a phenomenon. The attenuation in the channel between blades can be explained by the distortion of the planar waves by the mean flow, and can be calculated from the mean flow characteristics. The distortion of the waves at the outlet of the blade row is here evaluated by a Lagrangian tracking of particles seeded at the inlet. Figure 9-a shows the path-lines of the particles computed from the mean flow (the flow is quasi-steady and not turbulent in the channel between blades), whereas Fig. 9-b shows particle-lines seeded at the same time. Both plots show that the flow between the blades is not uniform in the azimuthal direction, and 
(a)

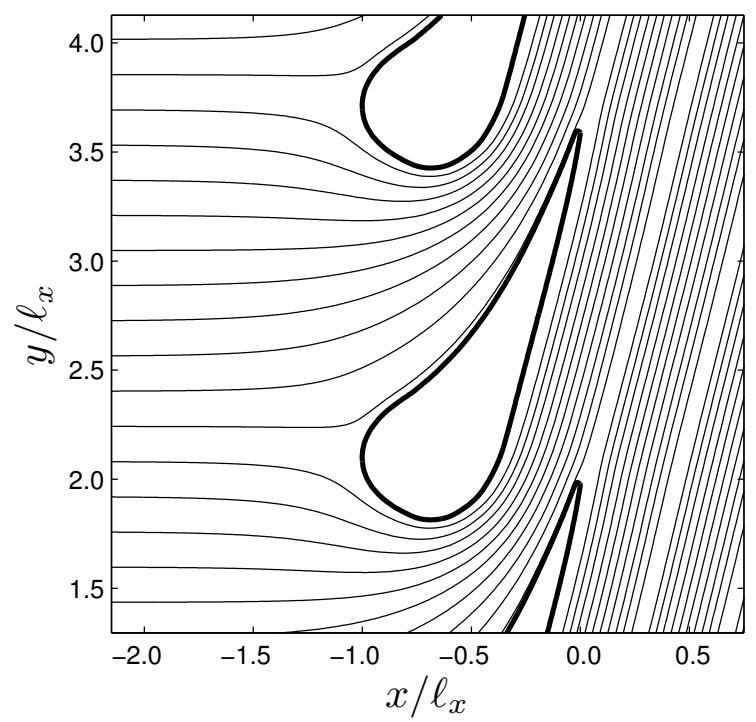

(b)

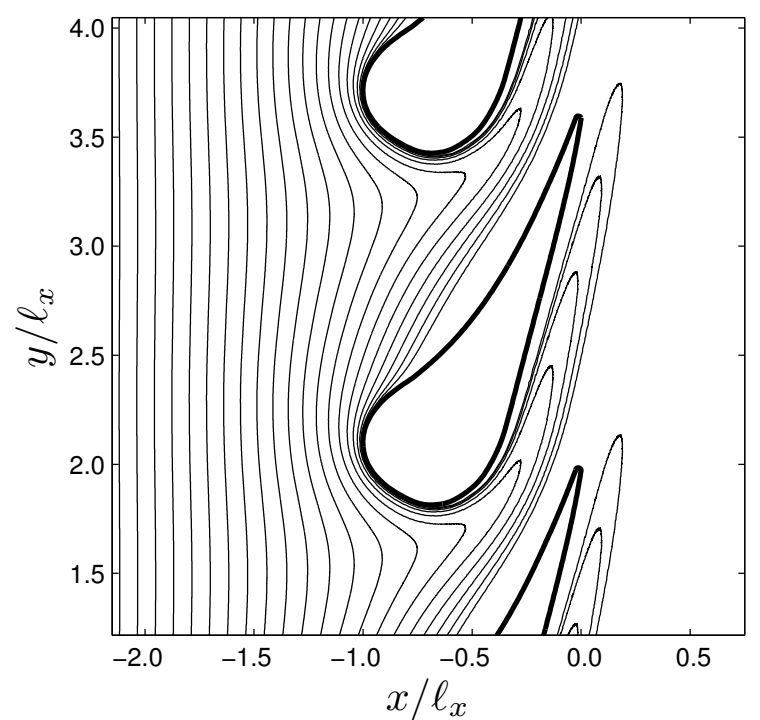

Figure 9: Mean flow characteristics. (a) Streamlines calculated from the mean flow; (b) Iso-temporal lines of massless particles seeded from the inlet and calculated from the mean flow.

therefore initially axially co-current particles have a different time of arrival at the blade row outlet plane $\left(x / l_{x}=0\right)$. Figure 9-b clearly reveals the deformation of the initially planar waves. The delay in the arrival of particles at the outlet $t_{d}(y)$ is plotted in Fig. 10-a versus the transversal direction $y$ normalized with the pitch length $L_{y}\left(y / L_{y}=0\right.$ and $y / L_{y}=1$ correspond to the blades trailing-edges). The particles passing by the trailing-edges should theoretically have an infinite time of arrival, but have a finite value here because of the finite number of path-lines used to evaluate the function $t_{d}(y)$. This function can be directly used to calculate the attenuation of the entropy waves, which no longer appear as planar at the outlet of the blade row. Indeed, each series of particles can be associated with a sinusoidal level of amplitude. As a consequence, the 1-D temporal fluctuations at the blade row outlet $d_{0}(t, k)$ can be simply expressed as follows:

$$
d_{0}(t, k)=\frac{1}{L_{y}} \int_{0}^{L_{y}} \exp \left\{i 2 \pi\left[k\left(t+t_{d}(y)\right) / \tau_{0}\right]\right\} d y
$$


(a)

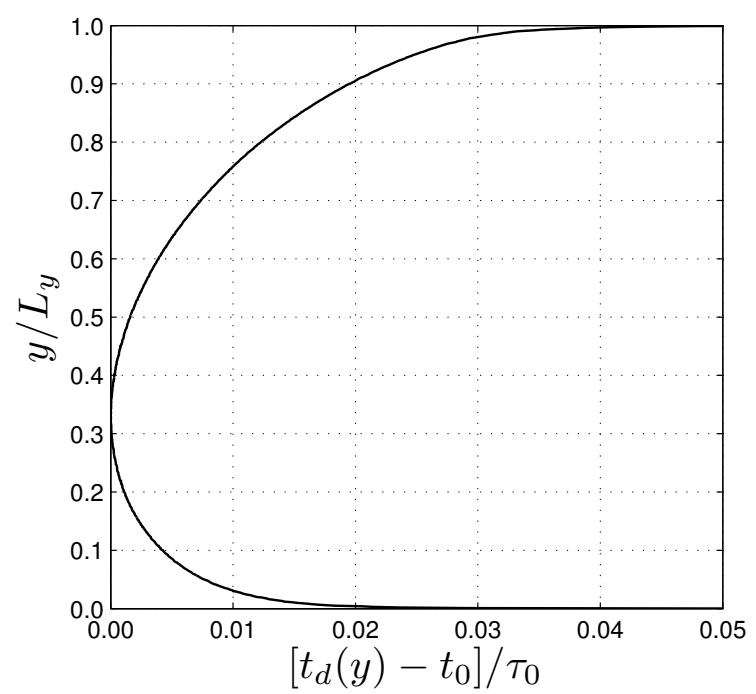

(b)

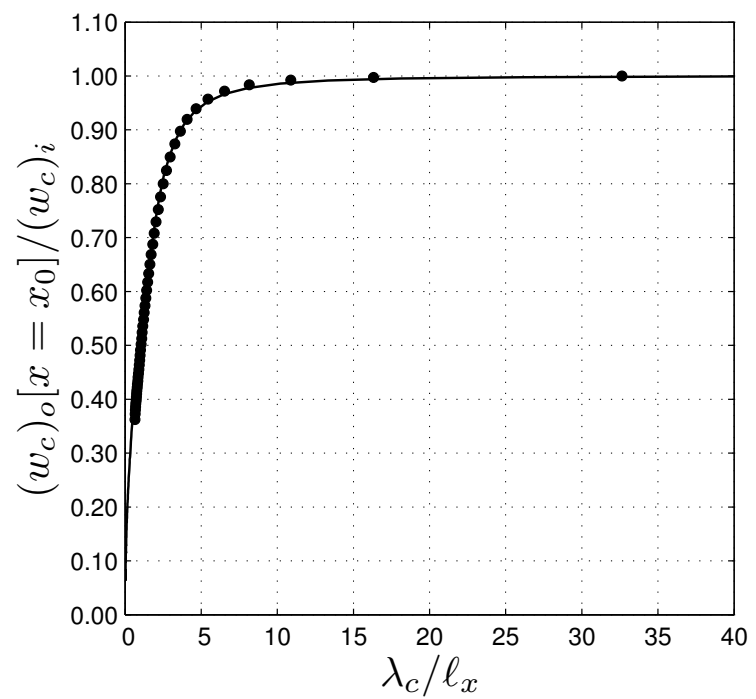

Figure 10: (a) Difference in time of arrival for a given curve to reach the blade row outlet $x_{0}$. (b) Attenuation of a convective wave ( $w_{c}$ ) through the blade row: calculated from the flow distortion $(-)$, simulated $(\bullet)$.

so that the corresponding attenuation $D_{0}(k)$ for the frequency $k$ is:

$$
D_{0}(k)=\left|\frac{1}{L_{y}} \int_{0}^{L_{y}} \exp \left\{i 2 \pi\left[k\left(t_{d}(y) / \tau_{0}\right)\right]\right\} d y\right|
$$

which depends on the function $t_{d}(y)$ and on $k$ only. Fig. 10-b shows that the attenuation measured in the simulation, and the one computed from the mean flow distortion, are in a quite good agreement for almost the whole spectrum. This strongly supports the idea that the attenuation for small convective wavelength at the beginning of the downstream flow is caused by the distortion of initially planar waves by the mean flow. This phenomenon will of course produce non-planar waves at the outlet. A more general expression of Eq. (46) can be:

$$
D_{m}(k)=\left|\frac{1}{L_{y}} \int_{0}^{L_{y}} \exp \left\{i 2 \pi\left[k\left(t_{d}(y) / \tau_{0}\right)+m\left(y / l_{y}\right)\right]\right\} d y\right|
$$

where the integer $m$ corresponds to the $m$-th transversal mode of propagation of the entropy wave. This function is plotted in Fig. 11 for different transversal modes. When the frequency tends to zero, only planar waves $(m=0)$ are 


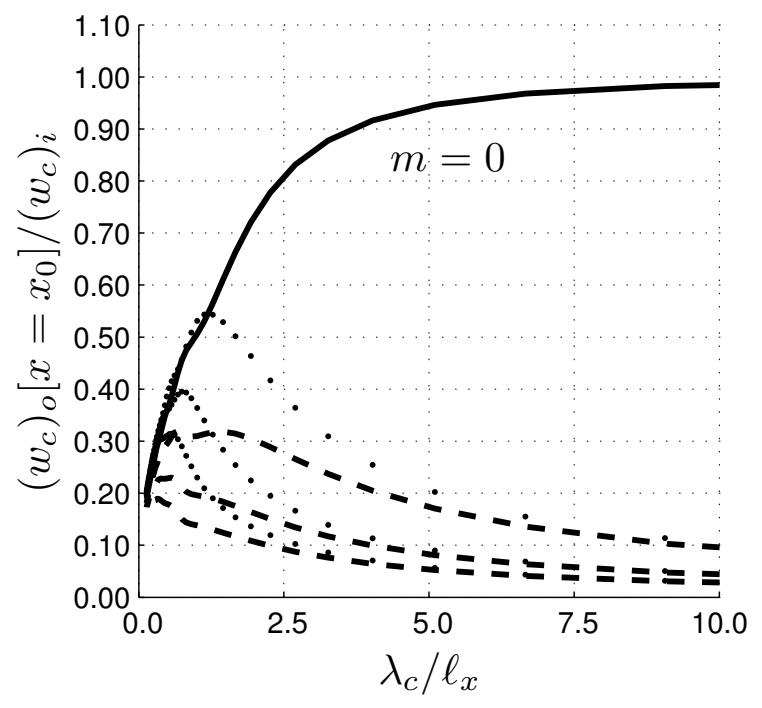

Figure 11: Attenuation of a convective wave $\left(w_{c}\right)$ through the blade row calculated from the flow distortion. Mode $m=0(-)$, positive modes $(---)$ and negative $(\cdots)$.

observed without attenuation. When the wavelength is close to the dimension of the blade, times $m$, the transversal modes are maximum, whereas the planar mode strongly decreases. These observations suggest that, to study noncompact aspects of the waves transmission and generation, not only planar waves should be looked at but also spinning modes, even if the excitation is planar. This redistribution can have a significant influence when several blade rows are involved.

The model of Cumpsty and Marble [6] also predicts the generation of vorticity waves. Indeed, both acoustic and entropy waves generate vorticity waves when propagated through the stator vane, as shown in Fig. 12. Even though there is no noise theoretically caused by vorticity in the stator vane case presently investigated (due to the $\left.\theta_{1}=0\right)$ this could become relevant when coupling different blade rows. It is therefore interesting to study the vorticity wave generated by both acoustic and entropy waves propagating. In Fig. 12 the vorticity waves generated by acoustic perturbations are correctly predicted for large wavelengths, while those generated by entropy perturbations are underestimated. This is caused by the low level of vorticity induced in this case, which is of the same order of magnitude 
(a)

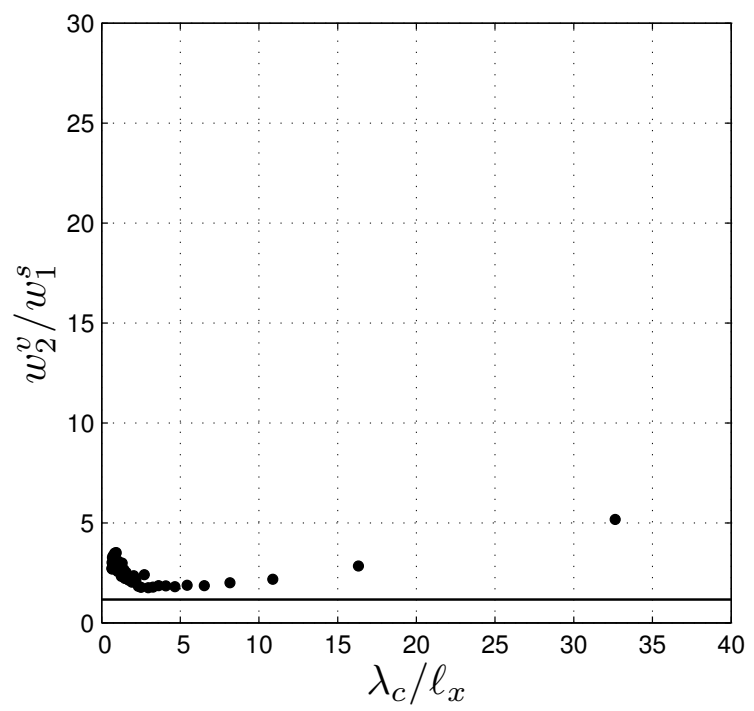

(b)

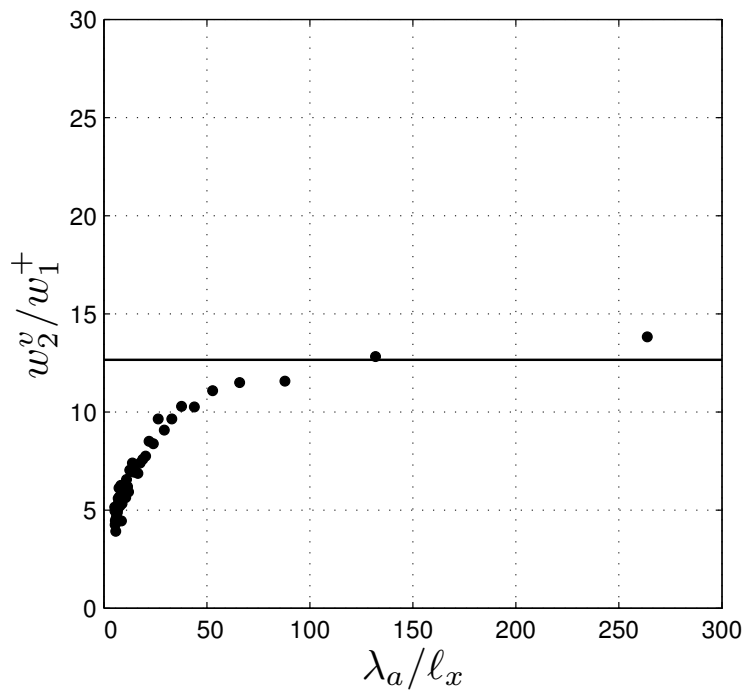

Figure 12: Vorticity response of the blade row to an entropy or acoustic perturbation at the inlet (cases S-1 and A-1). (a) Entropy; (b) Acoustic. Model (-), simulation (•).

as the vorticity present in the reference simulation $(\mathrm{N}-0)$ and due to the trailing-edge wake, as shown in Fig. 13 . The transfer functions for the vorticity wave (simulations V-1) are shown in Fig. 14 as a function of the non-dimensional convective wavelength. As seen in Appendix A, when the mean flow at the inlet of the blade row is straight $\left(\theta_{1}=0\right)$ vorticity waves generate no acoustic waves. The numerical simulations give a similar result for large wavelengths $\left(\lambda_{c} / \ell_{x}>5\right)$

Waves propagating downstream through the blade row generate both downstream and upstream propagating acoustic waves. When studying a complete turbine stage, the upstream propagating acoustic waves generated by the downstream blade rows have to be taken into account and correctly propagated. Simulation A-2 allows a comparison between the analytical model and the numerical simulations for the case of an upstream propagating acoustic wave through the blade row. Figure 15 shows the corresponding transfer functions, where it can be seen that at large wavelengths the simulation tends to the analytical value. 


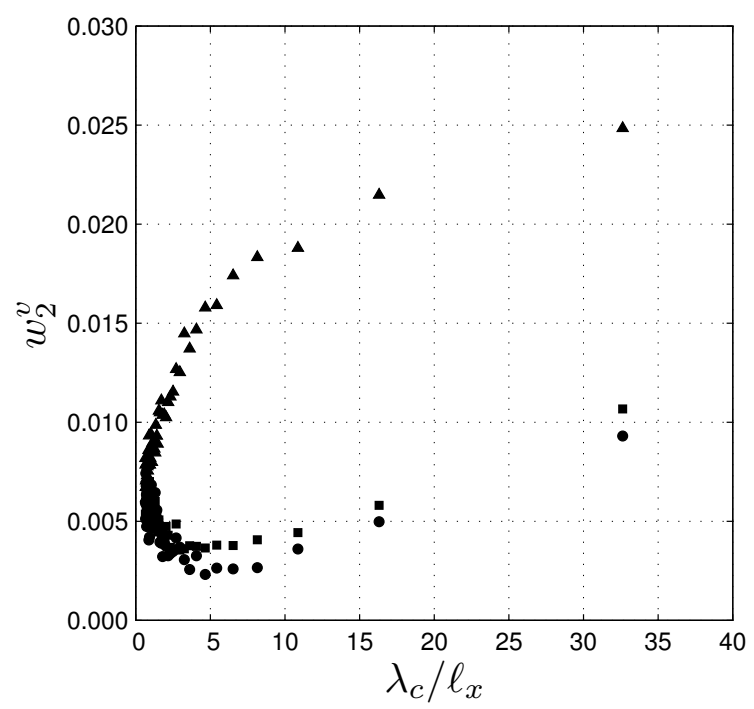

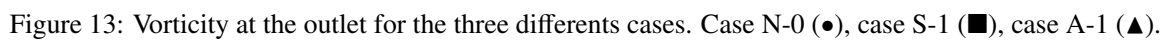

(a)

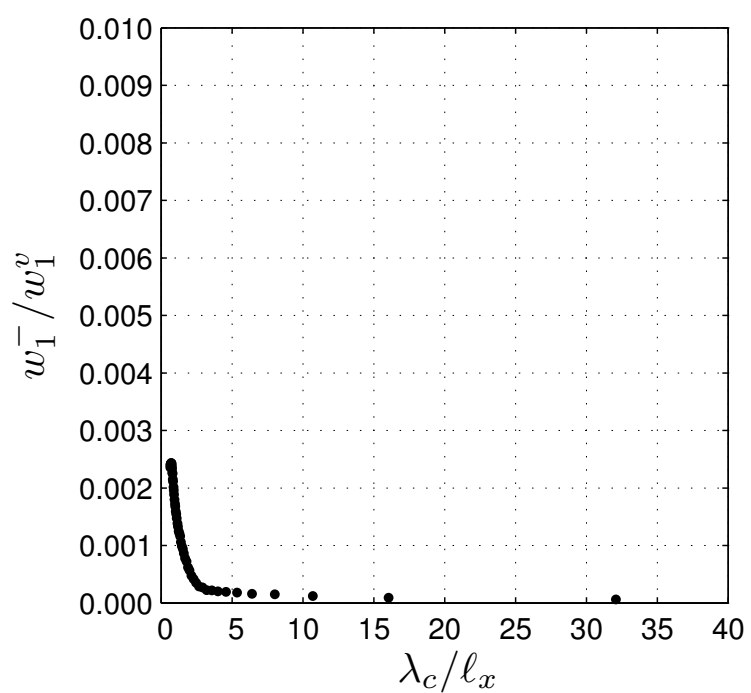

(b)

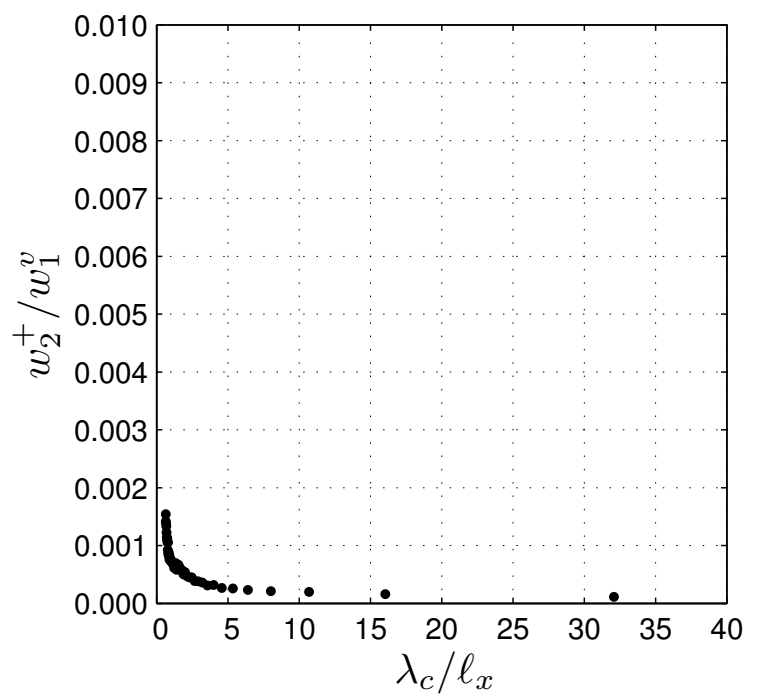

Figure 14: Acoustic response of the blade row to a vorticity perturbation at the inlet (case V-1). (a) Upstream propagating waves; (b) Downstream propagating waves. Model (-), simulation (•). 
(a)

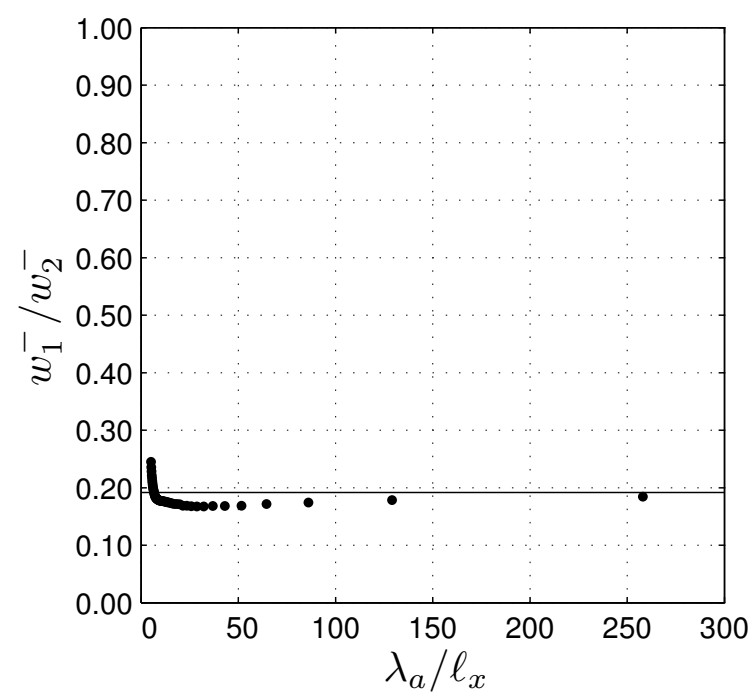

(b)

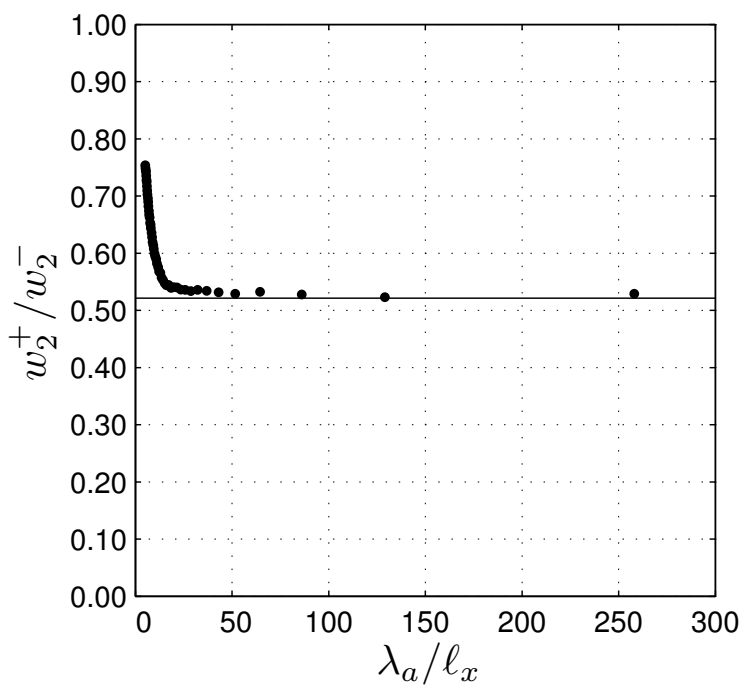

Figure 15: Acoustic response of the blade row to an acoustic perturbation at the outlet (case A-2). (a) Upstream propagating waves; (b) Downstream propagating waves. Model (-), simulation (•).

Overall, the transfer functions for all waves obtained with the simulations are in good agreement with the compact analytical model at low frequencies. This is not the case when the wavelength is of the order of the blade length, or smaller. However perturbations within a aeronautical combustion chamber are expected to be low frequency, so that this results have to be considered in the combustion-noise framework, that is to say taking into account the spectra of entropy and acoustic waves at the combustion chamber outlet. To calculate the errors made on the noise prediction by the use of the model on a real configuration the spectra of entropy and downward propagating acoustic wave, obtained with LES on a model SAFRAN combustor, are used (see Fig. 16), where it can be seen that the entropy wave at the outlet of the combustion chamber is much larger than the acoustic wave. The approach to evaluate this spectrum is the same as the one used throughout the article (planar waves and $k_{y}=0$ ). The noise power $(\mathrm{P})$ at the outlet is calculated as follows:

$$
P=\sqrt{\left.\int_{0}^{N_{k} / \tau_{0}}[T F S)\right]^{2} d f}
$$


(a)

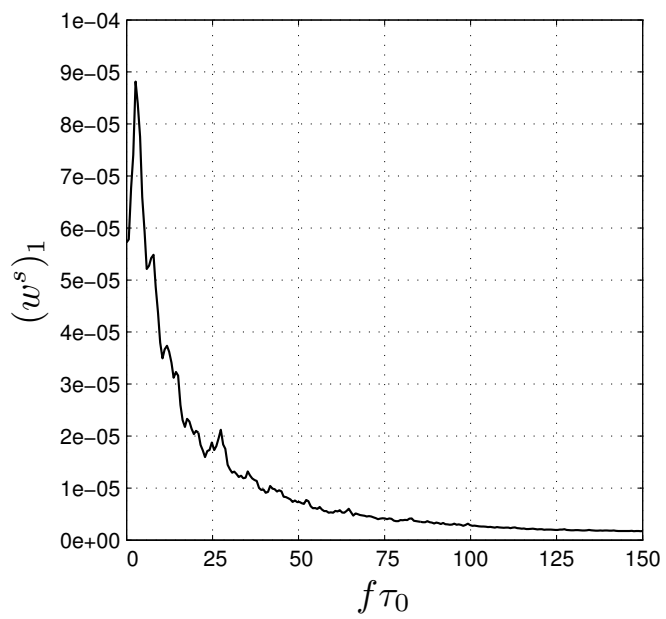

(b)

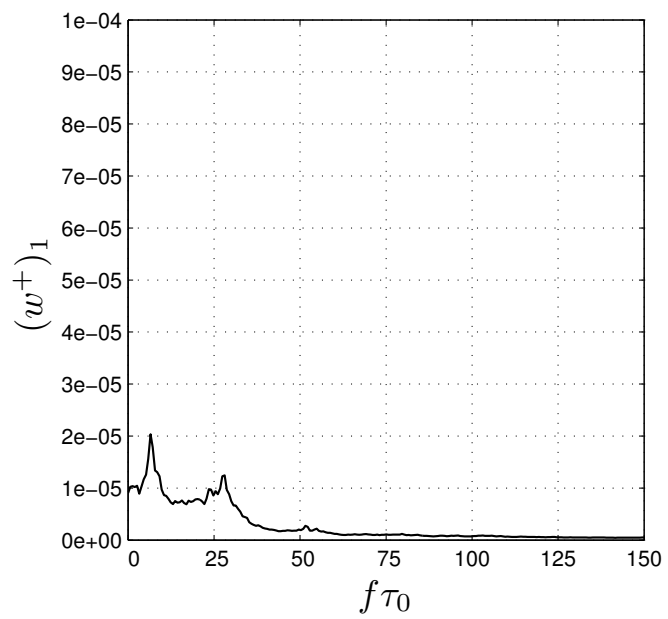

Figure 16: Spectra of sources from LES. (a) Entropy source; (b) Acoustic source.

$\mathrm{TF}$ is the transfer function (analytical with the compact assumption, or the one obtained with the simulations) and $\mathrm{S}$ is the source term shown in Fig. 16 (entropy for the indirect noise and acoustic for the direct noise). The noise for the acoustic (direct noise) is calculated with both transfer functions (TF): the one obtained by simulations and the one obtained with the analytical model. The error between the two methods is given by

$$
\epsilon \%=100 \times \frac{\left.P\right|_{\text {Analytical }}-\left.P\right|_{\text {Numerical }}}{\left.P\right|_{\text {Numerical }}}
$$

The result obtained by the model is found to be only $17 \%$ different from the real case when comparing the noise power as presented in Eq. (49), or equivalently $0.6 \mathrm{~dB}$. This error rises up to $56 \%$ for the entropy (indirect noise), or a $2 \mathrm{~dB}$ overestimate as reported in Tab. 2.

\section{Conclusions}

In the present study, the generalisation to a 2-D configuration made by Cumpsty and Marble [6] is shown to have a major effect on the acoustic transmission of both direct and indirect combustion noise through blade rows compared 


\begin{tabular}{lc}
\hline Name & Value \\
\hline Error from the model on Direct noise & $17 \%$ \\
Error from the model on Indirect noise & $56 \%$ \\
\hline Ratio Ind/Direct with model Transfer Function (TF) & 0.144 \\
Ratio Ind/Direct with real Transfer Function (TF) & 0.108 \\
\hline
\end{tabular}

Table 2: Errors due to the model when compared with the numerical simulation.

with the 1-D model of Marble and Candel [17], previously used to compare with the Entropy Wave Generator (EWG) experiment $[2,14]$. The 2-D model can deal with any angle of incidence of the impinging waves, and even for incident axial ones the difference can be large due to the strong deviation of the mean flow present in typical turbine blades. Moreover, the acoustic responses at the outlet of a blade row $w_{2}^{+}$to a purely axial entropy perturbation at the inlet $w_{1}^{s}$, and to an acoustic one at the inlet $w_{1}^{+}$are found to provide quite different results for important flow deviations as found in actual turbo-machines.

However, the 2-D analytical model still relies on the compact assumption that limits it to the low frequency range. Numerical unsteady simulations of the response of an isolated turbine stator vane have therefore been performed in a 2-D configuration to evaluate its range of validity and estimate the error made when applying the method to non-zero frequencies. The acoustic transmission and reflection are shown to be well predicted by the model in the frequency range corresponding to acoustic wavelengths $\lambda_{a} / \ell_{x}>10$. The acoustic waves generated by entropy disturbances impinging on the blade row (responsible for indirect noise) are also well predicted at low frequencies, corresponding to convective wavelengths $\lambda_{c} / \ell_{x}>10$, but the theoretical results rapidly depart from the numerical ones for higher frequencies. Another assumption in Cumpsty and Marble's [6] model is that entropy waves are just convected through the turbine blades with no distortion. Through a proper post-processing of the steady flow the initially planar entropy waves were shown to be strongly distorted by the inter-blade non-uniform steady flow of the high-pressure distributor. 
This occurs for entropy wavelengths smaller than the passage length, and therefore is significant at high frequencies. The distortion of the entropy wave scatters the initially plane wave into higher-order modes due to the azimuthal dependency of the mean flow velocity (not considered in the analytical 2-D model), reducing the amplitude of the plane mode. The attenuation of the plane mode due to this effect has been calculated in order to predict the decrease of indirect combustion noise at large frequencies. This phenomenon leads to additional disagreement with the 2-D model and can be significant when considering the multiple blade rows of a turbine. However, entropy perturbations within an actual aeronautical combustion chamber are expected to be mostly in the low-frequency range and this effect could be small. For this reason the derived transfer functions should be considered taking into account the acoustic and entropy waves as inputs coming from measurements or LES results at the exit of a combustion chamber. The calculation of the errors made on the noise prediction at the outlet of an actual distributor vane shows that an error of $0.6 \mathrm{~dB}$ is done on the direct noise, and $2.0 \mathrm{~dB}$ on the entropy one, when using the present model with disturbances spectra from an actual LES. The entropy fluctuations obtained in the latter are in the low-frequency range, therefore the overall indirect noises calculated analytically and numerically are in the same range of magnitude. The results provided by this first-order compact approach are globally quite good and make it suitable as part of a core-noise evaluation tool.

In this work only plane waves have been considered. These waves represent a large part of the contribution to combustion noise, as high-order azimuthal modes are cut-off at low frequencies. To completely validate the 2-D analytical model and its assumptions, azimuthal modes should be considered in a future work, as well as computing the transmission of waves through several blade rows, including rotor blades. 


\section{Appendix A.}

The analytical solutions of Eq. ((41)) are here provided for plane incoming waves. Considering $k_{y}=0$ and the Kutta condition given in Eq. (36), $\alpha=0$, Eq. ((40)) leads to following relationships:

$$
\begin{gathered}
w_{1}^{\mathrm{s}}=w_{2}^{\mathrm{s}} \\
-w_{1}^{\mathrm{s}}+w_{1}^{+}\left(1+\frac{1}{M_{1} \cos \theta_{1}}\right)+w_{1}^{-}\left(1-\frac{1}{M_{1} \cos \theta_{1}}\right)=\ldots \\
-w_{2}^{\mathrm{s}}+w_{2}^{+}\left(1+\frac{1}{M_{2} \cos \theta_{2}}\right)+w_{2}^{-}\left(1-\frac{1}{M_{2} \cos \theta_{2}}\right) \\
\mu_{1}\left[\frac{1}{\gamma-1} w_{1}^{\mathrm{s}}+w_{1}^{\mathrm{v}}\left(i M_{1}^{2} \cos \theta_{1} \sin \theta_{1}\right)+w_{1}^{+}\left(1+M_{1} \cos \theta_{1}\right)+w_{1}^{-}\left(1-M_{1} \cos \theta_{1}\right)\right]=\ldots \\
\mu_{2}\left[\frac{1}{\gamma-1} w_{2}^{\mathrm{s}}+w_{2}^{\mathrm{v}}\left(i M_{2}^{2} \cos \theta_{2} \sin \theta_{2}\right)+w_{2}^{+}\left(1+M_{2} \cos \theta_{2}\right)+w_{2}^{-}\left(1-M_{2} \cos \theta_{2}\right)\right] \\
i \cos ^{2} \theta_{2} w_{2}^{\mathrm{v}}+\frac{\sin \theta_{2}}{M_{2}}\left(w_{2}^{-}-w_{2}^{+}\right)=0
\end{gathered}
$$

where

$$
\mu=\frac{1}{1+\frac{1}{2}(\gamma-1) M^{2}}
$$

For incoming wave $w_{1}^{\mathrm{s}} \neq 0$ (other incoming waves are equal to zero):

$$
\begin{aligned}
& \frac{w_{1}^{-}}{w_{1}^{\mathrm{s}}}=\frac{\left(\frac{\mu_{1}}{\mu_{2}}-1\right) /(\gamma-1)}{\left(M_{1} \cos \theta_{1}-1\right)\left(\frac{\cos \theta_{2}+M_{2}}{\left(\cos \theta_{2}+1 / M_{2}\right) M_{1} \cos \theta_{1}}+\frac{\mu_{1}}{\mu_{2}}\right)} \\
& \frac{w_{2}^{+}}{w_{1}^{\mathrm{s}}}=\frac{1-\frac{1}{M_{1} \cos \theta_{1}} \frac{w_{1}^{-}}{w_{1}^{\mathrm{s}}}}{1+\frac{1}{M_{2} \cos \theta_{2}}} \\
& \frac{w_{2}^{\mathrm{v}}}{w_{1}^{\mathrm{s}}}=-i \frac{\sin \theta_{2}}{\cos ^{2} \theta_{2}} \frac{1}{M_{2}} \frac{w_{2}^{+}}{w_{1}^{\mathrm{s}}} \\
& \frac{w_{2}^{\mathrm{s}}}{w_{1}^{\mathrm{s}}}=1
\end{aligned}
$$


For incoming wave $w_{1}^{+} \neq 0$ (other incoming waves are equal to zero):

$$
\begin{aligned}
& \frac{w_{1}^{-}}{w_{1}^{+}}=-\frac{1+\frac{1}{M_{1} \cos \theta_{1}}-\frac{\mu_{1}}{\mu_{2}} \frac{\left(1+M_{1} \cos \theta_{1}\right)\left(M_{2} \cos \theta_{2}+1\right)}{M_{2}\left(\cos \theta_{2}+M_{2}\right)}}{1-\frac{1}{M_{1} \cos \theta_{1}}-\frac{\mu_{1}}{\mu_{2}} \frac{\left(1-M_{1} \cos \theta_{1}\right)\left(M_{2} \cos \theta_{2}+1\right)}{M_{2}\left(\cos \theta_{2}+M_{2}\right)}} \\
& \frac{w_{2}^{+}}{w_{1}^{+}}=\frac{\mu_{1}}{\mu_{2}} \frac{1+M_{1} \cos \theta_{1}+\frac{w_{1}^{-}}{w_{1}^{+}}}{1+\frac{M_{2}}{\cos \theta_{2}}} \\
& \frac{w_{2}^{\mathrm{v}}}{w_{1}^{+}}=-i \frac{\sin \theta_{2}}{\cos ^{2} \theta_{2}} \frac{1}{M_{2}} \frac{w_{2}^{+}}{w_{1}^{+}} \\
& \frac{w_{2}^{\mathrm{s}}}{w_{1}^{+}}=0
\end{aligned}
$$

For incoming wave $w_{2}^{-} \neq 0$ (other incoming waves are equal to zero):

$$
\begin{aligned}
& \frac{w_{1}^{-}}{w_{2}^{-}}=\frac{1-\frac{1}{M_{2} \cos \theta_{2}}-\frac{\left(\cos \theta_{2}-M_{2}\right)\left(M_{2} \cos \theta_{2}+1\right)}{M_{2}\left(\cos \theta_{2}+M_{2}\right) \cos \theta_{2}}}{1-\frac{1}{M_{1} \cos \theta_{1}}-\frac{\mu_{1}}{\mu_{2}} \frac{\left(1-M_{1} \cos \theta_{1}\right)\left(M_{2} \cos \theta_{2}+1\right)}{M_{2}\left(\cos \theta_{2}+M_{2}\right)}} \\
& \frac{w_{2}^{+}}{w_{2}^{-}}=\frac{\frac{\mu_{1}}{\mu_{2}}\left(1-M_{1} \cos \theta_{1}\right) \frac{w_{1}^{-}}{w_{2}^{-}}-\left(1-\frac{M_{2}}{\cos \theta_{2}}\right)}{1+\frac{M_{2}}{\cos \theta_{2}}} \\
& \frac{w_{2}^{\mathrm{v}}}{w_{2}^{-}}=+i \frac{\sin \theta_{2}}{\cos ^{2} \theta_{2}} \frac{1}{M_{2}}\left(1-\frac{w_{2}^{+}}{w_{2}^{-}}\right) \\
& \frac{w_{2}^{\mathrm{s}}}{w_{2}^{-}}=0
\end{aligned}
$$


For incoming wave $w_{1}^{\mathrm{v}} \neq 0$ (other incoming waves are equal to zero):

$$
\begin{aligned}
\frac{w_{1}^{-}}{w_{1}^{\mathrm{v}}} & =i \frac{\cos \theta_{1} \sin \theta_{1} M_{1}^{2}}{M_{1} \cos \theta_{1}-1+\frac{\mu_{2}}{\mu_{1}}\left(1+\frac{M_{2}}{\cos \theta_{2}}\right) \frac{1-1 / M_{1} \cos \theta_{1}}{1+1 / M_{1} \cos \theta_{1}}} \\
\frac{w_{2}^{+}}{w_{1}^{\mathrm{v}}} & =\frac{1-\frac{1}{M_{1} \cos \theta_{1}} \frac{w_{1}^{-}}{1+\frac{1}{M_{2} \cos \theta_{2}}}}{w_{1}^{\mathrm{v}}} \\
\frac{w_{2}^{\mathrm{v}}}{w_{1}^{\mathrm{v}}} & =-i \frac{\sin \theta_{2}}{\cos ^{2} \theta_{2}} \frac{1}{M_{2}} \frac{w_{2}^{+}}{w_{1}^{\mathrm{v}}} \\
\frac{w_{2}^{\mathrm{s}}}{w_{1}^{\mathrm{v}}} & =0
\end{aligned}
$$

\section{References}

1. F. Bake, N. Kings, A. Fischer, and I. Rohle. Experimental investigation of the entropy noise mechanism in aero-engines. International Journal of Aeroacoustics, 8(1):125-142, 2009.

2. F. Bake, C. Richter, B. Mühlbauer, N. Kings, I. Röhle, F. Thiele, and B. Noll. The entropy wave generator (ewg): A reference case on entropy noise. Journal of Sound and Vibration, 326(3-5):574-598, October 2009.

3. S. Candel. Analytical studies of some acoustic problems of jet engines. PhD thesis, California Institute of Technology, Pasadena, California, 1972.

4. L. Crocco. Supercritical gaseaous discharge with high frequency oscillations. Aerotechnica, 33(1):46-53, 1953.

5. N. Cumpsty and F. Marble. Core noise from gas turbine exhausts. J. Sound Vibration, 54(2):297-309, 1977.

6. N. Cumpsty and F. Marble. The interaction of entropy fluctuations with turbine blade rows; a mechanism of turbojet engine noise. Proc. R. Soc. Lond., 357(1690):323-344, Novembre 1977.

7. I. Duran, M. Leyko, S. Moreau, F. Nicoud, and T. Poinsot. Computing combustion noise by combining Large Eddy Simulations with analytical models for the propagation of waves through turbine blades. Comptes Rendus de l'Académie des Sciences - Mécanique, 341(1-2):131-140, January 2013.

8. I. Duran and S. Moreau. Solution of the quasi one-dimensional linearized Euler equations using flow invariants and the Magnus expansion. Journal of Fluid Mechanics, Accepted, 2013. 
9. I. Duran, S. Moreau, and T. Poinsot. Analytical and numerical study of combustion noise through a subsonic nozzle. AIAA Journal, 51(1):42$52,2013$.

10. J. E. Ffowcs Williams and M. S. Howe. The generation of sound by density inhomogeneities in low mach number nozzle flows. Journal of Fluid Mechanics, 70(3):605-622, 1975

11. C. S. Goh and A. S. Morgans. Phase prediction of the response of choked nozzles to entropy and acoustic disturbances. Journal of Sound and Vibration, pages 1-15, June 2011.

12. S. Kaji and T. Okazaki. Propagation of sound waves through a blade row: I. analysis based on the semi-actuator disk theory. Journal of Sound and Vibration, 11(3):339-353, March 1970.

13. S. Kaji and T. Okazaki. Propagation of sound waves through a blade row: II. analysis based on the acceleration potential method. Journal of Sound and Vibration, 11(3):355-375, March 1970.

14. M. Leyko, S. Moreau, F. Nicoud, and T. Poinsot. Numerical and analytical modelling of entropy noise in a supersonic nozzle with a shock. J. Sound Vib. , doi:10.1016/j.jsv.2011.01.025, 2011.

15. M. Leyko, F. Nicoud, S. Moreau, and T. Poinsot. Numerical and analytical investigation of the indirect combustion noise in a nozzle. Comptes Rendus Mécanique, 337(6-7):415-425, 2009.

16. M. Leyko, F. Nicoud, and T. Poinsot. Comparison of direct and indirect combustion noise mechanisms in a model combustor. AIAA Journal, 47(11):2709-2716, November 2009.

17. F. E. Marble and S. Candel. Acoustic disturbances from gas nonuniformities convected through a nozzle. J. Sound Vibration, 55(2):225-243, 1977.

18. A. Mishra and D. J. Bodony. Evaluation of actuator disk theory for predicting indirect combustion noise. Journal of Sound and Vibration, 332(4):821-838, February 2013.

19. V. Moureau, G. Lartigue, Y. Sommerer, C. Angelberger, O. Colin, and T. Poinsot. Numerical methods for unsteady compressible multicomponent reacting flows on fixed and moving grids. J. Comput. Phys., 202(2):710-736, 2005.

20. B. Mühlbauer, B. Noll, and M. Aigner. Numerical investigation of the fundamental mechanism for entropy noise generation in aero-engines. Acta Acustica united with Acustica, 95:470-478, 2009.

21. R. S. Muir. The application of a semi-actuator disk model to sound transmission calculations in turbomachinery, part I: The single blade row. Journal of Sound and Vibration, 54(3):393-408, October 1977.

22. R. S. Muir. The application of a semi-actuator disk model to sound transmission calculations in turbomachinery, part II: Multiple blade rows. Journal of Sound and Vibration, 55(3):335-349, Decembre 1977.

23. G. F. Pickett. Core engine noise due to temperature fluctuations convecting through turbine blade rows. In 2 nd AIAA Aeroacoustics Conference - AIAA 1975-528, 1975. 
24. H. Posson, S. Moreau, H. Blériot, B. de l'Epine, and C. Schram. Prediction of sound transmission through an annular cascade using an analytical cascade response function. In 16th AIAA/CEAS Aeroacoustics Conference and Exhibit, Stockholm, Sweden - AIAA Paper 2010$4030,2010$.

25. H. Posson and M. Roger. Parametric study of gust scattering and sound transmission through a blade row. In 13 th AIAA/CEAS Aeroacoustics Conference and Exhibit, Rome, Italy - AIAA Paper 2007-3690, 2007.

26. L. Quartapelle and V. Selmin. High-order Taylor-Galerkin methods for non-linear multidimensional problems., 1993.

27. S. R. Stow, A. P. Dowling, and T. P. Hynes. Reflection of circumferential modes in a choked nozzle. Journal of Fluid Mechanics, 467(1):215239, 2002.

28. H. S. Tsien. The transfer functions of rocket nozzles. Journal American Rocket Society, 22(3):139-143, May-June 1952. 